\title{
PLANAR LINKAGES FOLLOWING A PRESCRIBED MOTION
}

\author{
MATTEO GALLET ${ }^{*}, \circ$, CHRISTOPH KOUTSCHAN*, ZIJIA LI*, \\ GEORG REGENSBURGER ${ }^{\dagger}$, JOSEF SCHICHO, AND NELLY VILLAMIZAR
}

\begin{abstract}
Designing mechanical devices, called linkages, that draw a given plane curve has been a topic that interested engineers and mathematicians for hundreds of years, and recently also computer scientists. Already in 1876, Kempe proposed a procedure for solving the problem in full generality, but his constructions tend to be extremely complicated. We provide a novel algorithm that produces much simpler linkages, but works only for parametric curves. Our approach is to transform the problem into a factorization task over some noncommutative algebra. We show how to compute such a factorization, and how to use it to construct a linkage tracing a given curve.
\end{abstract}

\section{INTRODUCTION}

Kempe's Universality Theorem [14, stating that any plane algebraic curve can be drawn by a mechanical linkage with only rotational joints, surprised his contemporaries: his work represented a major breakthrough in a topic investigated by mathematicians of the 19th century. In the middle of the last century, it appeared in textbooks, for example, the ones by Lebesgue 20, and Blaschke and Müller 22. Recently, there was a revived interest in the problem among mathematicians and computer scientists as we outline in the following. We would also like to mention 22 for a historical overview and further references.

In modern terms, the procedure proposed by Kempe is a parsing algorithm. It takes the defining polynomial of a plane curve as input and realizes arithmetic operations via certain elementary linkages. In this work, we approach the question from a different perspective. Instead of a polynomial, we start with a rational parametrization of a curve. From this, we obtain a parametrized family of elements of the group $\mathrm{SE}_{2}$ of direct isometries of the plane, namely a motion, whose action on a point traces the given curve. In order to realize such a motion by a linkage, we decompose it into a series of revolutions. By encoding motions via polynomials over a noncommutative algebra, we reduce this task to a factorization problem. Eventually, we design a linkage whose rotational joints move according to the previously obtained revolutions, yielding a device drawing the desired curve.

Requiring a rational parametrization, it is clear that our approach is less general than Kempe's, and its subsequent generalizations [1, 12, 13, 15], because it cannot

2010 Mathematics Subject Classification. Primary 70B15, 68W30, 70G55, 20G20, 16Z05, 14P05, $12 \mathrm{Y} 05$.

First published in Mathematics of Computation in 2016, published by American Mathematical Society.

* Supported by the Austrian Science Fund (FWF): W1214.

- Supported by the Austrian Science Fund (FWF): P26607 - "Algebraic Methods in Kinematics: Motion Factorisation and Bond Theory".

$\dagger$ Supported by the Austrian Science Fund (FWF): P27229. 
be applied to planar curves of positive genus. However, the parametric setup has advantages in design processes: in the language of robotics/kinematics, we are not just prescribing the position of the end effector but also its orientation, in a similar way as in [25]; moreover, when one has to interpolate prescribed poses of the end effector, we can specify a tracing order and have control over its speed.

Linkages obtained via Kempe's procedure do not, in general, trace only the curve they are designed for. More precisely, the curve is only drawn by a component of the configuration space (also called workspace), that is, the set of all positions reachable by the linkage. This is due to the fact that already the elementary linkages may admit degenerate configurations, allowing the device to flip into an unwanted component. Several solutions to this problem have been suggested [1, 6, 12, 13, 15]. The linkages produced by our method present the same issue, however, this can be treated using the techniques presented in [1, 6]; see Remark 7.7.

Generation of linkages drawing an arbitrary curve may involve many links and joints. This was already observed by Kempe [14]: "... this method would not be practically useful on account of the complexity of the link work employed...". The problem of finding upper bounds for the number of joints needed to trace a given planar curve of degree $d$ was first addressed in [7, 8]; see [6, Section 3.2.3]. Their upper bound $\mathrm{O}\left(d^{4}\right)$ was later improved by Abbott and Barton to $\mathrm{O}\left(d^{2}\right)$, who in [1] showed that this bound is optimal. In this paper, we provide an algorithm that applies to rational planar curves: given a rational parametrization with denominator of degree $d$ and without real roots, our algorithm produces a linkage with $3 d+2$ links and $\frac{9}{2} d+1$ joints (Proposition 7.10, we are grateful to Hans-Peter Schröcker for discussions leading to this result). If we apply our technique to the parametrization of an ellipse, we obtain a linkage with 8 links and 10 joints; in Section 2 , we illustrate our approach and the main ideas of our paper via this example. In contrast, an unoptimized linkage returned by Kempe's procedure has 158 links and 235 joints; we are grateful to Alexander Kobel, who gave a full implementation [16] of Kempe's procedure, for assisting us in the computation of these numbers.

In Section 3, we develop a mathematical model for linkages with only rotational joints. In particular, we define their configuration space in terms of isometries sending a fixed initial configuration to a reachable one. This differs from the commonly used models [5, 12, 13, 15, 24] which, instead, define configurations in terms of the positions of links and joints.

In Section 4, we recall that one can embed $\mathrm{SE}_{2}$ as an open subset of a real projective space; see [11. This allows us to introduce a noncommutative algebra $\mathbb{K}$ whose multiplication corresponds to the group operation in $\mathrm{SE}_{2}$, hence mimicking the role played by dual quaternions with respect to $\mathrm{SE}_{3}$. A polynomial with coefficients in $\mathbb{K}$ therefore describes a family of direct isometries, which we call a rational motion. Consequently, we refer to such polynomials as motion polynomials; they are the two-dimensional analog of the motion polynomials introduced in [10, and they are the key for turning our geometric problem into an algebraic one. In fact, we show that a linear motion polynomial represents a motion constrained by a revolute or prismatic joint. Hence a factorization of a motion polynomial into linear factors yields a decomposition of the corresponding rational motion into simple ones.

In Section 5, we give necessary and sufficient criteria for the existence of a factorization of a motion polynomial. Not every motion polynomial admits such a 
factorization. However, the correspondence between motion polynomials and rational motions is not one-to-one: for every rational motion, there is a whole equivalence class of motion polynomials. Moreover, the motions we are dealing with are special, since they admit bounded orbits. Once we restrict to such bounded rational motions, we can prove that the equivalence class of every bounded rational motion contains a motion polynomial admitting a factorization into linear polynomials of revolute type. We provide an algorithm for computing the smallest factorizable polynomial in an equivalence class and one of its factorizations; see Theorem 5.15 . In this context, we would like to refer to the preprint [21, where this result is used to prove factorizability of motion polynomials giving rational motions in $\mathrm{SE}_{3}$.

At the beginning of Section 7. we construct from a factorization a linkage, in the form of an open chain, such that the given rational motion can be realized as the relative motion of the last link of the chain with respect to the first one. Open chains have high mobility, so we have to constrain our linkage such that it performs only the motion we are interested in. The technique we employ, called flip procedure, is introduced in Section 6. In the remaining part of Section 7, we exploit the properties of flips and propose our main algorithm; see Theorem 7.6 .

In Section 8, we address the problem of self-collisions. For arbitrary linkages, this is a challenging problem [5. Section 9.3]. Here, for the first time in the paper, we take into account how linkages are physically realized. We show that self-collisions can be efficiently detected for linkages obtained by our algorithm when links are realized by bars. If, instead, we allow links of different shapes, we describe a construction showing that it is possible to realize these linkages without collisions. This addresses the open problems raised in [6, Open Problem 3.2] and [23, Section 2.3].

A popular formulation of Kempe's Theorem states that "There is a linkage that signs your name". Inspired by this, in Section 9, we give an example of a linkage drawing a calligraphic letter.

All algorithms described in the paper have been implemented by the secondnamed author in the computer algebra system Mathematica. The source code, an expository notebook, and animations for the main examples are available for free as electronic supplementary material [17].

This paper is the outcome of the joint work of the Symbolic Computation group at RICAM (Linz): the problem of constructing linkages following a planar rational motion was proposed in our research seminar and after some time became its main topic. Each participant contributed to the final result according to his/her own background and skills (algebraic geometry, combinatorics, kinematics, etc).

\section{A first EXAMPLE}

We want to illustrate the main ideas and techniques with an example. Consider the ellipse $(x+1)^{2}+4 y^{2}=1$ in the plane, which admits the rational parametrization

$$
\varphi(t)=\left(\frac{-2}{t^{2}+1}, \frac{t}{t^{2}+1}\right) .
$$

Our goal is to construct a planar linkage with rotational joints drawing the curve parametrized by $\varphi$ that admits only one degree of freedom. This means that there is a specific link (on which we put the pen) that performs a motion along the ellipse as the linkage moves. By construction, the motion of this link is the composition of several rotations. We encode motions by univariate polynomials with coefficients 
in the algebra $\mathbb{K}=\mathbb{C}[\eta] /\left(\eta^{2}, \imath \eta+\eta \imath\right)$, where $\imath$ denotes the imaginary unit. Elements of $\mathbb{K}$ are of the form $z+\eta w$ with $z, w \in \mathbb{C}$, and they are multiplied as follows:

$$
(z+\eta w) \cdot\left(z^{\prime}+\eta w^{\prime}\right)=\left(z z^{\prime}\right)+\eta\left(\bar{z} w^{\prime}+z^{\prime} w\right) .
$$

In our case, a translational motion along the ellipse is represented by the polynomial

$$
P(t)=\left(t^{2}+1\right)+\eta(\imath t-2) .
$$

This means that the orbit of any point under this motion is a translate of the ellipse. To construct a linkage that realizes this motion, we want to factor $P$ into linear polynomials, which correspond to revolute motions. However, one can check that this is not at all possible! On the other hand, as pointed out in Remark 4.8. we will see that for any $R \in \mathbb{R}[t]$, the motion polynomial $R P \in \mathbb{K}[t]$ describes the same motion as $P$. In the example, we can take $R=t^{2}+1$ so that $R P$ admits a factorization into linear polynomials (see Theorem 5.15 ):

$$
R(t) \cdot P(t)=(t+\imath-\eta \imath) \cdot\left(t-\imath+\frac{1}{2} \eta \imath\right) \cdot\left(t-\imath+\frac{3}{2} \eta \imath\right) \cdot(t+\imath) .
$$

Such a factorization is computed by the algorithm FactorMotionPolynomial (see the end of Section 5p. It allows us to construct a linkage, in the form of an open chain (left part of Figure 1), whose links move according to the rotations represented by the linear factors (this is algorithm ConstructWeakLinkage at the beginning of Section 7). Since such a linkage has a high degree of freedom, we need to constrain its mobility by adding more links and joints (right part of Figure 1). This is achieved by an iteration of the so-called flip procedure, described in Sections 6 and 7 .

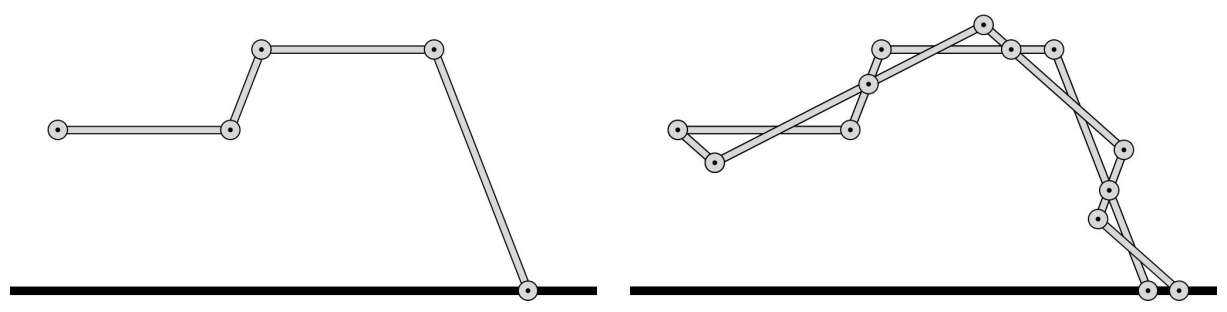

Figure 1. An open chain and its extension to a linkage of mobility one, realizing the translational motion given by $P(t)$.

However, if we just want to draw the ellipse, we need not realize exactly the translational motion $P(t)$ : it is enough to find a motion for which the orbit of one point is the ellipse. As pointed out in Remark 4.9, multiplying with a polynomial $C \in \mathbb{C}[t]$ from the left does not change the orbit of the origin. In our case, we find that the polynomial $C P$ with $C(t)=t-\imath$ factors completely as follows (see Proposition 7.10:

$$
C(t) \cdot P(t)=\left(t-\imath-\frac{1}{2} \eta \imath\right) \cdot\left(t-\imath+\frac{1}{2} \eta \imath\right) \cdot(t+\imath+\eta \imath) .
$$

This factorization gives rise to a slightly simpler construction, see Figure 2, Further details concerning this example will be provided in Examples 4.12, 5.16, and 7.11. 


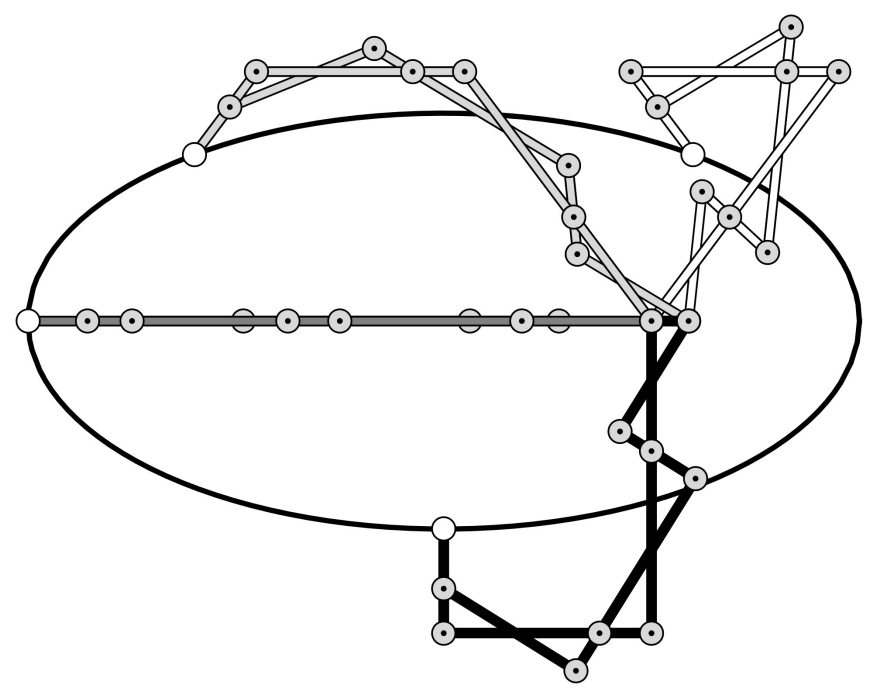

Figure 2. The linkage that draws an ellipse. The same linkage is shown in different positions: $t=2$ (white), $t=\frac{1}{2}$ (light gray), $t=0$ (dark gray), and $t=-1$ (black).

\section{LinKAGES}

In this section, we define a mathematical model for kinematic objects known as linkages. A linkage is a device constituted by rigid bodies, called links, connected by joints, which restrict the relative position of two neighboring links. In this paper we focus on planar linkages with revolute joints, namely linkages for which all links move in parallel planes and whose joints allow only rotations around a point. In our model we will not be concerned with the shape of the links - the only exception will be Section 8 - and we will suppose that joints are the only constraints for the motion of the links. Thus we can represent a linkage by a graph whose vertices correspond to links, and where two vertices are connected by an edge if and only if the corresponding links are connected by a joint (for an example, see Figure 3). But the graph does not encode how the joints constrain the motion of the links, since it does not specify their (initial) position. So we have to add this extra information.

Definition 3.1. A linkage with revolute joints is a connected undirected graph $G=(V, E)$, together with a map $\rho: E \longrightarrow \mathbb{R}^{2}$, such that $G$ does not have selfloops, i.e., all edges connect different vertices. The elements of $V$ are called links, while the elements of $E$ are called joints. We call two links neighboring if they are connected by a joint. For a joint $e \in E$, the point $\rho(e)$ is called the center of rotation of $e$. In the following, we will always assume that $V$ is of the form $\{1, \ldots, n\}$ and that elements of $E$ are given by unordered pairs $\{i, j\}$ of elements $i, j \in V$.

Remark 3.2. An implementation of a linkage, where links are realized by line segments between the joints, also looks like a graph. Note that this graph is not the same as the one in Definition 3.1, rather it is its dual. The lengths of the links are then given implicitly by distances between initial positions of joints. 

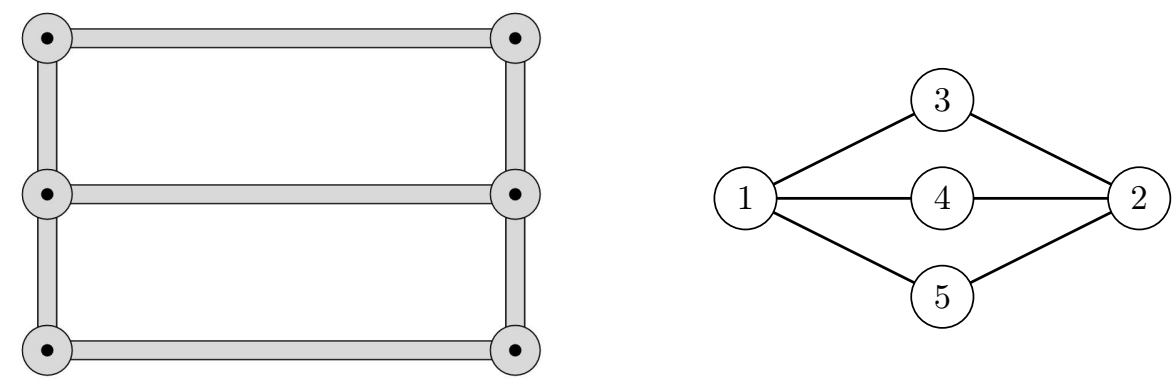

Figure 3. A picture of a linkage (on the left) and the corresponding link graph (on the right).

Next we explain how we describe configurations of a linkage, namely, the positions of all its links at a given moment. There are essentially two ways to indicate the position of a link: we can give either its absolute position with respect to some frame of reference, or its relative position with respect to some other link. Both these notions will be encoded by means of isometries.

Notation. We denote by $\mathrm{SE}_{2}$ the group of direct isometries of $\mathbb{R}^{2}$, i.e., maps that preserve distances and orientation (of the standard basis of $\mathbb{R}^{2}$ ):

$$
\mathrm{SE}_{2}:=\left\{\sigma: \mathbb{R}^{2} \longrightarrow \mathbb{R}^{2}: \sigma \text { is an isometry, } \operatorname{det} \sigma=1\right\} .
$$

For each link $i$ there is a unique isometry $\sigma_{i} \in \mathrm{SE}_{2}$ taking it from its initial position to a current one; we say that $\sigma_{i}$ is the absolute position of the link $i$. The relative position of a link $i$ with respect to some other link $j$ is defined to be $\sigma_{i, j}=\sigma_{i} \circ \sigma_{j}^{-1}$; hence we have the relation $\sigma_{i, j}=\sigma_{i, k} \circ \sigma_{k, j}$. If $i$ and $j$ are neighboring links, then $\sigma_{i, j}$ is a rotation around the point $p=\rho(i, j)$, which is the initial position of the joint $\{i, j\}$. This follows directly, since $\sigma_{i}(p)=\sigma_{j}(p)$. All this motivates the following definition of "virtual" relative positions; the name indicates that they do not take into account the constraints imposed by the linkage.

Definition 3.3. Let $L=(G, \rho)$ be a linkage, and let $i, j \in V$ be links connected by a joint $e \in E$. The set of virtual relative positions $\operatorname{VRP}(i, j)$ of the link $i$ with respect to the link $j$ is the subgroup of $\mathrm{SE}_{2}$ of rotations around the point $\rho(e)$. Notice that $\operatorname{VRP}(i, j)=\operatorname{VRP}(j, i)$.

We define a configuration of a linkage $L$ as a collection of virtual relative positions of links satisfying the following conditions: if $\left(i, h_{1}\right),\left(h_{1}, h_{2}\right), \ldots,\left(h_{s}, i\right)$ is a directed cycle in $G$, namely a sequence of pairs of links connected by a joint, starting and ending at the same link, then the composition $\sigma_{i, h_{1}} \circ \cdots \circ \sigma_{h_{s}, i}$ - which gives the relative position of $i$ with respect to itself - should be the identity. Loosely speaking, a configuration consists of the angles at all joints (counted twice), together with the (redundant) information $\rho(e)$ for all $e \in E$. 
Definition 3.4. The set of configurations of a linkage $L$ is defined to be

$$
\begin{aligned}
\operatorname{Conf}(L):= & \left\{\left(\sigma_{k, l}\right) \in \prod_{\{i, j\} \in E} \operatorname{VRP}(i, j) \times \operatorname{VRP}(j, i):\right. \text { for every directed cycle } \\
& \left.\left(i, h_{1}\right),\left(h_{1}, h_{2}\right), \ldots,\left(h_{s}, i\right) \text { in } G, \text { we have } \sigma_{i, h_{1}} \circ \cdots \circ \sigma_{h_{s}, i}=\mathrm{id}\right\},
\end{aligned}
$$

where $\prod$ denotes the Cartesian product so that $\left(\sigma_{k, l}\right)$ is a tuple of size $2|E|$.

This definition of configuration space is the main difference between our model and the ones appearing in the literature [5, 12, 13, 15, 24.

Remark 3.5. The cycle conditions imposed in the definition of $\operatorname{Conf}(L)$ have the following consequence: suppose that $i, j \in V$ are two neighboring links; then $(i, j),(j, i)$ is a directed cycle from $i$ to $i$. Therefore, if $\Sigma=\left(\sigma_{k, l}\right)$ is a configuration of $L$, the cycle condition imposes that $\sigma_{i, j} \circ \sigma_{j, i}=\mathrm{id}$, implying that $\sigma_{i, j}=\sigma_{j, i}^{-1}$. Hence for every linkage $L=(G, \rho)$, the projection

$$
\prod_{\{i, j\} \in E} \operatorname{VRP}(i, j) \times \operatorname{VRP}(j, i) \longrightarrow \prod_{\substack{\{i, j\} \in E \\ i<j}} \operatorname{VRP}(i, j)
$$

is a bijection when restricted to $\operatorname{Conf}(L)$ (and an isomorphism if we consider the projective structure on $\operatorname{Conf}(L)$ we will define soon), and similarly for every projection that forgets exactly one among each pair $(i, j)$ and $(j, i)$. Still, we chose the above definition for $\operatorname{Conf}(L)$ because it does not fix an orientation of the edges, allowing us to deal with arbitrary directed paths (see Definition 3.7).

Notice that for every pair of neighboring links $i$ and $j$, the subgroup $\operatorname{VRP}(i, j)$ can be set-theoretically identified with the real projective line $\mathbb{P}_{\mathbb{R}}^{1}$. Under this identification, every cycle condition imposed in Definition 3.4 becomes a closed condition in the Zariski topology, since it is given by multihomogeneous polynomials. In this way $\operatorname{Conf}(L)$ acquires the structure of a projective subvariety of $\left(\mathbb{P}_{\mathbb{R}}^{1}\right)^{2|E|}$.

Definition 3.6. We define the mobility of a linkage $L$ to be the dimension of the configuration space $\operatorname{Conf}(L)$ as a projective subvariety of $\left(\mathbb{P}_{\mathbb{R}}^{1}\right)^{2|E|}$.

So far we only took into account the relative position of two neighboring links. For our purposes, namely to construct a linkage that follows a prescribed motion, we need to take one link (the "base") as fixed, and consider the relative positions of all the other links with respect to the base.

Definition 3.7. Let $L=(G, \rho)$ be a linkage. Let $\Sigma \in \operatorname{Conf}(L)$ and let $i, j \in V$ be links. Let $\left(i, h_{1}\right),\left(h_{1}, h_{2}\right), \ldots,\left(h_{s}, j\right)$ be a directed path in $G$ from $i$ to $j$ - which exists, since by Definition 3.1 the graph $G$ is connected. Then we define the relative position of $j$ with respect to $i$ in the configuration $\Sigma=\left(\sigma_{k, l}\right)$ as

$$
\mathrm{RP}(i, j, \Sigma):=\sigma_{i, h_{1}} \circ \cdots \circ \sigma_{h_{s}, j} \in \mathrm{SE}_{2} .
$$

Notice that, because of the cycle condition, this definition is independent of the chosen path. We define the set of relative positions of $j$ with respect to $i$ to be

$$
\operatorname{RP}(i, j):=\{\operatorname{RP}(i, j, \Sigma): \Sigma \in \operatorname{Conf}(L)\} \subseteq \mathrm{SE}_{2} .
$$




\section{Motion POLYNOMials}

As mentioned in Section 1, our goal is to reduce the main problem to an algebraic one. We first introduce an algebraic setting for manipulating isometries, consisting in a noncommutative $\mathbb{R}$-algebra $\mathbb{K}$ whose multiplication corresponds to the group operation in $\mathrm{SE}_{2}$. This is an instance of a general construction associating a Clifford algebra to each group of isometries $\mathrm{SE}_{n}$; see for example [26, Section 9.2]. Then we define the main object of our work, motion polynomials, as polynomials over $\mathbb{K}$.

Following [11, one can embed $\mathrm{SE}_{2}$ in the real projective space $\mathbb{P}_{\mathbb{R}}^{3}$ with coordinates $x_{1}, x_{2}, y_{1}, y_{2}$, as the open subset

$$
U:=\mathbb{P}_{\mathbb{R}}^{3} \backslash\left\{\left(x_{1}: x_{2}: y_{1}: y_{2}\right): x_{1}^{2}+x_{2}^{2}=0\right\} .
$$

Hence $U$ is the complement of the projective line $x_{1}=x_{2}=0$. The (right) action of an element $\left(x_{1}: x_{2}: y_{1}: y_{2}\right) \in U$ on a point $(x, y) \in \mathbb{R}^{2}$ is given by:

$$
\left(\begin{array}{l}
x \\
y
\end{array}\right) \mapsto \frac{1}{x_{1}^{2}+x_{2}^{2}}\left[\left(\begin{array}{cc}
x_{1}^{2}-x_{2}^{2} & -2 x_{1} x_{2} \\
2 x_{1} x_{2} & x_{1}^{2}-x_{2}^{2}
\end{array}\right)\left(\begin{array}{l}
x \\
y
\end{array}\right)+\left(\begin{array}{l}
x_{1} y_{1}-x_{2} y_{2} \\
x_{1} y_{2}+x_{2} y_{1}
\end{array}\right)\right] .
$$

Moreover, the group operation in $\mathrm{SE}_{2}$ becomes a bilinear map:

$$
\begin{aligned}
\left(x_{1}: x_{2}: y_{1}: y_{2}\right) \cdot\left(x_{1}^{\prime}: x_{2}^{\prime}: y_{1}^{\prime}: y_{2}^{\prime}\right)= & \left(x_{1} x_{1}^{\prime}-x_{2} x_{2}^{\prime}: x_{1} x_{2}^{\prime}+x_{2} x_{1}^{\prime}:\right. \\
& x_{1} y_{1}^{\prime}+x_{2} y_{2}^{\prime}+y_{1} x_{1}^{\prime}-y_{2} x_{2}^{\prime}: \\
& \left.x_{1} y_{2}^{\prime}-x_{2} y_{1}^{\prime}+y_{1} x_{2}^{\prime}+y_{2} x_{1}^{\prime}\right),
\end{aligned}
$$

where $\left(x_{1}: x_{2}: y_{1}: y_{2}\right)$ and $\left(x_{1}^{\prime}: x_{2}^{\prime}: y_{1}^{\prime}: y_{2}^{\prime}\right)$ represent two direct isometries $\sigma, \sigma^{\prime} \in \mathrm{SE}_{2}$, respectively.

For an easier handling of the multiplication in this embedding of $\mathrm{SE}_{2}$, we introduce the following notation: we write a representative $\left(x_{1}: x_{2}: y_{1}: y_{2}\right)$ of an element of $\mathrm{SE}_{2}$ as a pair $(z, w) \in \mathbb{C}^{2}$, where $z=x_{1}+\imath x_{2}$ and $w=y_{1}+\imath y_{2}$ (here $\imath$ is the imaginary unit). Then Equation (4.3) can be rewritten concisely as

$$
(z, w) \cdot\left(z^{\prime}, w^{\prime}\right)=\left(z z^{\prime}, \bar{z} w^{\prime}+z^{\prime} w\right)
$$

where $z, z^{\prime}, w$ and $w^{\prime}$ are multiplied as complex numbers, and the bar $\overline{(\cdot)}$ is complex conjugation. We can go further, by writing a pair $(z, w)$ in the form $z+\eta w$, and by postulating that $\eta$ satisfies the two relations:

$$
z \eta=\eta \bar{z} \text { for all } z \in \mathbb{C} \text { and } \eta^{2}=0 .
$$

Then the multiplication we obtain is exactly the one described in Equation (4.4):

$$
(z+\eta w) \cdot\left(z^{\prime}+\eta w^{\prime}\right)=z z^{\prime}+\eta\left(\bar{z} w^{\prime}+z^{\prime} w\right) .
$$

Definition 4.1. We define the $\mathbb{R}$-algebra

$$
\mathbb{K}:=\mathbb{C}[\eta] /\left(\eta^{2}, \imath \eta+\eta \imath\right) .
$$

Based on the previous discussions, we can identify elements of $\mathbb{K}$ with elements of $\mathrm{SE}_{2}$. In this way the projective space $\mathbb{P}_{\mathbb{R}}^{3}$ in which we embed $\mathrm{SE}_{2}$ can be thought as the projectivization $\mathbb{P}(\mathbb{K})$ of $\mathbb{K}$, considered as an $\mathbb{R}$-vector space.

Notice that the algebra $\mathbb{K}$ is constructed in such a way that its multiplication is a lift of the group operation of $\mathrm{SE}_{2}$ : this means that if two isometries $\sigma_{1}, \sigma_{2} \in \mathrm{SE}_{2}$ are represented by $k_{1}, k_{2} \in \mathbb{K}$, then $\sigma_{2} \circ \sigma_{1}$ is represented by $k_{1} \cdot k_{2}$ (remember we have a right action). More precisely, one can prove that $\mathbb{K}$ is isomorphic to the even subalgebra $\mathcal{C} \ell^{+}(0,2,1)$ of the Clifford algebra $\mathcal{C} \ell(0,2,1)$ of $\mathrm{SE}_{2}$; see [26, Section 9.2]. 
Remark 4.2. Looking at the construction of the algebra $\mathbb{K}$, we notice that the condition $x_{1}^{2}+x_{2}^{2} \neq 0$ for points in $\mathbb{P}_{\mathbb{R}}^{3}$ representing isometries in $\mathrm{SE}_{2}$ becomes $z \neq 0$ when we consider elements of $\mathbb{K}$. Moreover, the identity isometry is represented in $\mathbb{P}_{\mathbb{R}}^{3}$ by the point $(1: 0: 0: 0)$, hence by any purely real element of $\mathbb{K}$, namely elements of the form $z+\eta w$ with $z \in \mathbb{R}$ and $w=0$. From this we see that, given $k=z+\eta w \in \mathbb{K}$ representing an isometry $\sigma \in \mathrm{SE}_{2}$, then $k^{\prime}=\bar{z}-\eta w$ represents $\sigma^{-1}$, since the product $k k^{\prime}$ equals $|z|^{2}$, which is purely real.

The following lemma characterizes simple subgroups of $\mathrm{SE}_{2}$ in a geometric fashion: it shows that both translational and revolute motions, i.e., families of translations resp. rotations, correspond to lines in $\mathbb{P}_{\mathbb{R}}^{3}$.

Lemma 4.3. Let $\ell \subseteq \mathbb{P}_{\mathbb{R}}^{3}$ be a projective line passing through the point $(1: 0: 0: 0)$, and define $\ell_{U}=\ell \cap U$ and $X=\ell \backslash U$, where $U$ is defined in Equation 4.1. Then:

(i) if $X$ has cardinality 1 , then $\ell_{U}$ corresponds to a subgroup of $\mathrm{SE}_{2}$ that consists of all translations along a fixed common direction;

(ii) if $X$ is empty, then $\ell_{U}$ corresponds to a subgroup of $\mathrm{SE}_{2}$ that consists of all rotations around a fixed common point.

Proof. We analyze the two cases separately.

$A d(i)$ : By hypothesis we have that $(1: 0: 0: 0) \in \ell$ and $(0: 0: a: b) \in X \subset \ell$ for some $a, b \in \mathbb{R}$, not both zero. Hence the line $\ell$ admits the parametrization:

$$
(\lambda: 0: a \mu: b \mu), \quad \text { for }(\lambda: \mu) \in \mathbb{P}_{\mathbb{R}}^{1} .
$$

Plugging this parametrization into Equation 4.2 , we see that the elements of $\ell_{U}$ are translations by the vector $\mu / \lambda(a, b)$.

$A d($ (ii): Again by hypothesis we have that $(1: 0: 0: 0) \in \ell$; moreover there exists a point in $\ell$ which is of the form $(0: a: b: c)$. Since $X$ is assumed to be empty, we have $a \neq 0$, and hence the line $\ell$ admits the parametrization:

$$
(\lambda: a \mu: b \mu: c \mu), \quad \text { for }(\lambda: \mu) \in \mathbb{P}_{\mathbb{R}}^{1} .
$$

We use Equation 4.2 to compute the fixed points of an arbitrary element of $\ell$. A direct calculation reveals that we always get the point $(-c / 2 a, b / 2 a)$, which is independent of $\lambda$ and $\mu$. Thus every point of $\ell_{U}$ represents a rotation around it.

The description of revolutions around a point given by Lemma 4.3 enables us to compute effectively the configuration curve of a linkage, as shown in Example 4.4

Example 4.4. Let us consider the linkage $L$ whose graph is depicted in Figure 4 The linkage $L$ is given by 4 links and 4 joints, and the map $\rho$ is determined by:

$$
\begin{aligned}
& \rho(1,3)=u_{1}=(0,-3 / 2), \\
& \rho(3,4)=u_{3}=(-3 / 4,9 / 2), \\
& \rho(2,4)=u_{4}=(-1,13 / 2), \\
& \rho(1,2)=u_{2}=(-1 / 4,1 / 2) .
\end{aligned}
$$

We compute the configuration set of $L$ and its dimension as a projective variety. Since we have 4 joints, it will be a projective subvariety of $\left(\mathbb{P}_{\mathbb{R}}^{1}\right)^{4}$ (here, rather than $\operatorname{Conf}(L)$, we are considering one of its projections, but as noticed in Remark 3.5 we get an isomorphic object). Lemma 4.3 ensures that the 4 subgroups of $\mathrm{SE}_{2}$ of rotations around the points $u_{i}$ - which constitute the subgroups of virtual positions 


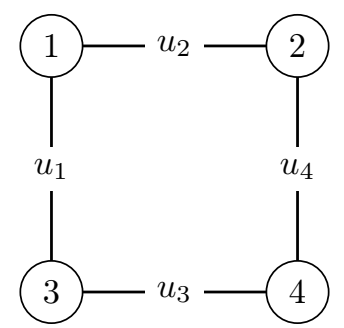

Figure 4. Graph of a linkage constituted by 4 links and 4 joints, a so-called closed $4 R$-linkage. The edges are labeled by points $u_{i}$ in $\mathbb{R}^{2}$ that are the images of the edges under the map $\rho$.

between two neighboring links as in Definition 3.3 - correspond to 4 lines $\ell_{i} \subseteq \mathbb{P}_{\mathbb{R}}^{3}$ with parametrizations:

\begin{tabular}{cc} 
center $u_{i}$ & parametrization of line $\ell_{i}$ \\
\hline$(0,-3 / 2)$ & $\left(\lambda_{1}: \mu_{1}:-3 \mu_{1}: 0\right)$ \\
$(-3 / 4,9 / 2)$ & $\left(\lambda_{3}: 2 \mu_{3}: 18 \mu_{3}: 3 \mu_{3}\right)$ \\
$(-1,13 / 2)$ & $\left(\lambda_{4}: \mu_{4}: 13 \mu_{4}: 2 \mu_{4}\right)$ \\
$(-1 / 4,1 / 2)$ & $\left(\lambda_{2}: 2 \mu_{2}: 2 \mu_{2}: \mu_{2}\right)$
\end{tabular}

where $\left(\lambda_{i}: \mu_{i}\right) \in \mathbb{P}_{\mathbb{R}}^{1}$ for all $i \in\{1, \ldots, 4\}$. Recall from Definition 3.4 that, in this case, a configuration for $L$ is a 4 -tuple $\left(\sigma_{1,2}, \sigma_{2,3}, \sigma_{3,4}, \sigma_{4,1}\right)$ of direct isometries that satisfies the cycle condition $\sigma_{1,2} \circ \sigma_{2,3} \circ \sigma_{3,4} \circ \sigma_{4,1}=$ id. Each of the isometries $\sigma_{k, l}$ gives a point on one projective line $\ell_{i}$, and in our projective model of $\mathrm{SE}_{2}$ composition corresponds to multiplication according to Equation 4.3 . Under these identifications, the composition $\sigma_{1,2} \circ \sigma_{2,3} \circ \sigma_{3,4} \circ \sigma_{4,1}$ corresponds to a point $\left(F_{1}: F_{2}: F_{3}: F_{4}\right) \in \mathbb{P}_{\mathbb{R}}^{3}$, where all $F_{i}$ are polynomials in the variables $\left(\lambda_{1}: \mu_{1}\right), \ldots,\left(\lambda_{4}: \mu_{4}\right)$. Noticing that the identity element of $\mathrm{SE}_{2}$ is represented by the point $(1: 0: 0: 0) \in \mathbb{P}_{\mathbb{R}}^{3}$, one realizes that the cycle condition is equivalent to

$$
\operatorname{rk}\left(\begin{array}{cccc}
F_{1} & F_{2} & F_{3} & F_{4} \\
1 & 0 & 0 & 0
\end{array}\right)=1 .
$$

Hence, as a subvariety of $\left(\mathbb{P}_{\mathbb{R}}^{1}\right)^{4}$, the configuration set $\operatorname{Conf}(L)$ is the zero set of the polynomials $F_{2}, F_{3}, F_{4}$. A computer algebra computation shows that this is a one-dimensional variety with two components.

We introduce now one of the main concepts of this paper, namely motion polynomials. Intuitively, a motion can be described as a curve in the space of direct isometries. In our case, we want such a curve to be defined by a rational parametrization.

Definition 4.5. Let $X_{1}, X_{2}, Y_{1}, Y_{2} \in \mathbb{R}[t]$ be polynomials such that $X_{1}^{2}+X_{2}^{2}$ is not identically zero; denote by $V\left(X_{1}, X_{2}, Y_{1}, Y_{2}\right)$ the set of their common zeros in $\mathbb{R}$. The map $\phi: \mathbb{R} \backslash V\left(X_{1}, X_{2}, Y_{1}, Y_{2}\right) \longrightarrow \mathbb{P}_{\mathbb{R}}^{3}$ defined by $X_{1}, X_{2}, Y_{1}, Y_{2}$ is called a rational motion. We will use the notation $\phi: \mathbb{R} \rightarrow \mathbb{P}_{\mathbb{R}}^{3}$ to mean that $\phi$ is not defined everywhere on $\mathbb{R}$. Thinking of $\mathrm{SE}_{2}$ as an open subset of $\mathbb{P}_{\mathbb{R}}^{3}$, the condition on $X_{1}^{2}+X_{2}^{2}$ ensures that for all but finitely many $t \in \mathbb{R}$ we have $\phi(t) \in \mathrm{SE}_{2}$.

Definition 4.6. Let $\phi$ be a rational motion given by $\left(X_{1}, X_{2}, Y_{1}, Y_{2}\right)$. The polynomial $P(t)=Z(t)+\eta W(t) \in \mathbb{K}[t]$, where $Z=X_{1}+\imath X_{2}$ and $W=Y_{1}+\imath Y_{2}$, is called 
a motion polynomial encoding the motion $\phi$. The polynomials $Z$ and $W$ are respectively called the primal and secondary part of $P$, denoted by $\operatorname{pp}(P)$ resp. $\operatorname{sp}(P)$.

Next we want to connect motion polynomials with rational plane curves. For this purpose it is helpful to rephrase the action of $\mathrm{SE}_{2}$ on $\mathbb{R}^{2}$ shown in Equation $(4.2)$ in the following way: we identify a point $(x, y) \in \mathbb{R}^{2}$ with the element $u=x+\imath y \in \mathbb{C}$; if $\sigma \in \mathrm{SE}_{2}$ is represented by an element $z+\eta w \in \mathbb{K}$, then $\sigma$ sends $u$ to

$$
\frac{u z^{2}+z w}{|z|^{2}}
$$

Using this formulation of the (right) action, it is easy to prove the following result.

Proposition 4.7. Let $\varphi: \mathbb{R} \longrightarrow \mathbb{R}^{2}$ be a rational parametrization of a real curve, which means that $\varphi$ is of the form

$$
\varphi(t)=\left(\frac{f(t)}{h(t)}, \frac{g(t)}{h(t)}\right)
$$

for some real polynomials $f, g$ and $h$. Then the orbit of the origin under the motion given by the motion polynomial $P=h+\eta(f+\imath g)$ is exactly the image of $\varphi$.

Let $P_{1}$ and $P_{2}$ be two motion polynomials. Because of the algebraic properties of $\mathbb{K}$, the polynomial $P=P_{1} P_{2}$ defines a motion that is the composition of the motions determined by $P_{2}$ and $P_{1}$. More precisely, for every $t \in \mathbb{R}$, the isometry $P(t)$ is the composition of the isometries $P_{2}(t)$ and $P_{1}(t)$. This shows how important the factorization of a motion polynomial is in our framework: it provides a decomposition of a motion into simpler ones.

We saw in Lemma 4.3 that rational motions whose image is a line in $\mathbb{P}_{\mathbb{R}}^{3}$ are translational motions or revolutions. Hence linear motion polynomials encode this kind of motions. It follows that, if we are able to factor a motion polynomial into linear ones, then we can decompose a rational motion into revolutions and translational motions. Later we will consider a suitable subclass of motion polynomials, so that only the first situation occurs.

Remark 4.8. Let $\phi: \mathbb{R} \rightarrow \mathbb{P}_{\mathbb{R}}^{3}$ be a rational motion and let $P(t) \in \mathbb{K}[t]$ be the corresponding motion polynomial, so $P=Z+\eta W$ with $Z, W \in \mathbb{C}[t]$. Notice that, although $Z$ and $W$ have coefficients in $\mathbb{C}$, the polynomial $P$ determines a real curve in $\mathbb{P}_{\mathbb{R}}^{3}$, namely a curve described by real equations. Hence, if $R \in \mathbb{R}[t]$ is a nonzero real polynomial, one can check that $R P \in \mathbb{K}[t]$ provides the same curve in $\mathbb{P}_{\mathbb{R}}^{3}$ as $P$.

Remark 4.9. Let again $\phi: \mathbb{R} \rightarrow \mathbb{P}_{\mathbb{R}}^{3}$ be a rational motion and let $P(t) \in \mathbb{K}[t]$ be the corresponding motion polynomial. For any $C \in \mathbb{C}[t]$ the orbits of the origin under the motions given by $P$ and $C P$ are the same since $C$ represents a revolution around the origin - recall that $C P$ acts on $\mathbb{R}^{2}$ by first applying $C$ and then $P$.

Example 4.10. The rational motion given by the polynomial $t+\eta$ is a vertical translational motion. Indeed, its image in $\mathbb{P}_{\mathbb{R}}^{3}$ is parametrized by $(\lambda: 0: \mu: 0)$, so that we are in case (i) of Lemma 4.3 In contrast, the motion polynomial $P(t)=$ $t+\imath$ gives a revolution around the origin $(0,0)$. This is an instance of case (ii) of Lemma 4.3 using the parametrization $(\lambda: \mu: 0: 0)$.

Example 4.11. Consider the following product of two linear motion polynomials:

$$
\underbrace{(t+\imath)}_{\begin{array}{c}
\text { rotation around } \\
\text { a point }
\end{array}} \cdot \underbrace{(t-\imath+\eta)}_{\begin{array}{c}
\text { rotation in the opposite } \\
\text { sense, around another point }
\end{array}}=\underbrace{\left(t^{2}+1\right)+\eta(t-\imath)}_{\begin{array}{c}
\text { this is a translation, since } \\
\text { there is no imaginary part in } t^{2}+1
\end{array}}
$$


For a fixed $t$ we get a translation by the vector $\frac{1}{t^{2}+1}(t,-1)$. As $t$ changes, this vector describes a circle, hence we get a translational motion along a circle (see Figure 5).

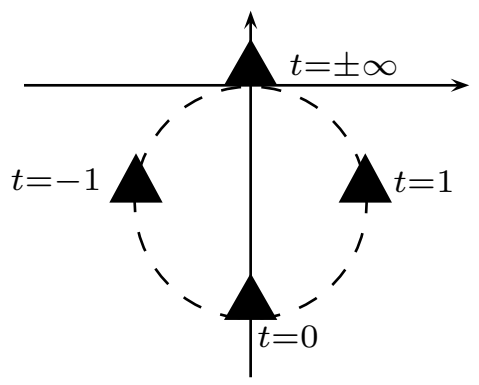

Figure 5. The trace of the point $(0,0)$ under the circular translation given by the motion polynomial $\left(t^{2}+1\right)+\eta(t-\imath)$ is depicted via a dashed line. The action of the motion on the black triangle highlights the fact that we have a purely translational motion.

In Example 4.11 we saw that the factorization of a motion polynomial into linear polynomials provides a decomposition of the described motion into revolutions. However, Example 4.12 shows that this is not always possible.

Example 4.12. Let us consider an elliptic translational motion:

$$
P(t)=\left(t^{2}+1\right)+\eta(a t-b \imath), \quad a, b \in \mathbb{R} .
$$

We try to factor $P$ into two linear polynomials $P_{1}, P_{2} \in \mathbb{K}[t]$. First notice that the primal parts of $P_{1}$ and $P_{2}$ should be factors of $\left(t^{2}+1\right)$, so they have to be of the form $t \pm \imath$. Thus we have only two possibilities:

$$
\left\{\begin{array} { l } 
{ \operatorname { p p } ( P _ { 1 } ) = t + \imath } \\
{ \operatorname { p p } ( P _ { 2 } ) = t - \imath }
\end{array} \quad \left\{\begin{array}{l}
\operatorname{pp}\left(P_{1}\right)=t-\imath \\
\operatorname{pp}\left(P_{2}\right)=t+\imath
\end{array}\right.\right.
$$

By a direct computation one can prove that none of those two choices gives a factorization if $a \neq b$, thus $P$ cannot be factored into linear polynomials.

On the other hand, we show now that if we multiply $P$ by a real polynomial $R$ we can achieve a factorization. Recall that, by Remark 4.8 the polynomials $P$ and $R P$ describe the same motion. In this case we take $R=t^{2}+1$. Hence we need four linear polynomials $P_{1}, \ldots, P_{4}$ to factorize $R P$, and again $\operatorname{pp}\left(P_{i}\right)=t \pm \imath$. We make the following ansatz:

$$
\begin{array}{ll}
P_{1}=(t-\imath)+\eta w_{1}, & P_{3}=(t+\imath)+\eta w_{3}, \\
P_{2}=(t+\imath)+\eta w_{2}, & P_{4}=(t-\imath)+\eta w_{4} .
\end{array}
$$

Imposing $P_{1} \cdots P_{4}=R P$ gives a linear system in the $w_{i}$, namely:

$$
\left\{\begin{array}{l}
w_{1}+w_{2}+w_{3}+w_{4}=a \\
w_{1}+w_{2}-w_{3}-w_{4}=b
\end{array}\right.
$$

Each solution of the system 4.7) gives rise to a factorization of the polynomial $R P$. If, instead, we take the following ansatz:

$$
\begin{array}{ll}
P_{1}=(t+\imath)+\eta w_{1}, & P_{3}=(t+\imath)+\eta w_{3}, \\
P_{2}=(t-\imath)+\eta w_{2}, & P_{4}=(t-\imath)+\eta w_{4} .
\end{array}
$$

one can check that the corresponding linear system does not admit any solution. 
In the next section, we consider the factorization problem in a more systematic way. We will see that, once we restrict to a certain class of motion polynomials, all situations can be treated as we did in Example 4.12 in general a motion polynomial $P$ cannot be factored into linear polynomials in $\mathbb{K}[t]$, but it is possible to find a real polynomial $R$ such that $R P$ can be factored.

We end this section by defining precisely what we mean by saying that a linkage realizes a given rational motion. Recall from Definition 3.7 that, given two links $i$ and $j$ of a linkage $L$, we can define the set $\operatorname{RP}(i, j)$ of relative positions of the link $j$ with respect to the link $i$, and this is a subset of $\mathrm{SE}_{2}$. Furthermore, recall that the set $\operatorname{Conf}(L)$ of configurations of $L$ has the structure of a real projective variety. We are going to use some concepts and results coming from real algebraic geometry:

(i) every projective space $\mathbb{P}_{\mathbb{R}}^{n}$ is a real affine variety, namely there exists an embedding $\xi: \mathbb{P}_{\mathbb{R}}^{n} \hookrightarrow \mathbb{R}^{k}$ for some $k \in \mathbb{N}$, and $\xi\left(\mathbb{P}_{\mathbb{R}}^{n}\right)$ is the zero set of a collection of real polynomials; see for example [3, Theorem 3.4.4];

(ii) a subset $X \subseteq \mathbb{R}^{k}$ is called semialgebraic if it can be described as the set of points satisfying a disjunction of conjunctions of polynomial equalities and inequalities; see for example [3, Definition 2.1.4];

(iii) a subset $X \subseteq \mathbb{P}_{\mathbb{R}}^{n}$ is called semialgebraic if its image under any embedding $\xi: \mathbb{P}_{\mathbb{R}}^{n} \hookrightarrow \mathbb{R}^{k}$ as in (i) is a semialgebraic set in the sense of (ii).

Lemma 4.13. Let $L$ be a linkage and let $i, j$ be links of $L$. Then, under the projective embedding of $\mathrm{SE}_{2}$, the set $\mathrm{RP}(i, j)$ becomes a semialgebraic subset of $\mathrm{SE}_{2}$.

Proof. Let $\left(i, h_{1}\right), \ldots,\left(h_{s}, j\right)$ be any directed path from $i$ to $j$. Consider the map $F: \operatorname{Conf}(L) \longrightarrow \mathrm{SE}_{2}$ sending a configuration $\Sigma=\left(\sigma_{k, l}\right)$ to $\sigma_{i, h_{1}} \circ \cdots \circ \sigma_{h_{s}, j}$. One can check that $\operatorname{RP}(i, j)$ coincides with $F(\operatorname{Conf}(L))$. Moreover, if we write the map $F$ in terms of the projective coordinates of $\operatorname{Conf}(L)$ and $\mathrm{SE}_{2}$, then $F$ is given by real polynomials. Hence, because of a general result in real algebraic geometry (see for example [3, Theorem 1.4.2]), the set $\operatorname{RP}(i, j)$ is semialgebraic.

In the following definition, and later in this paper, the word "component" will always stand for "irreducible component" with respect to the Zariski topology.

Definition 4.14. Let $L$ be a linkage and let $\phi: \mathbb{R} \rightarrow \mathbb{P}_{\mathbb{R}}^{3}$ be a rational motion. We say that

(i) $L$ weakly realizes the motion $\phi$ if there exist links $i$ and $j$ of $L$ such that image $(\phi) \subseteq \mathrm{RP}(i, j)$.

(ii) $L$ strongly realizes the motion $\phi$ if there exist links $i$ and $j$ of $L$ such that image $(\phi) \subseteq \operatorname{RP}(i, j)$ and $\overline{\text { image }(\phi)}$ is a component of $\operatorname{RP}(i, j)$, where $\overline{(\cdot)}$ denotes the Zariski closure in $\mathrm{RP}(i, j)$.

Remark 4.15. Notice that in case (ii) of Definition 4.14 we allow the given variety of relative positions to have several components, and we ask that only one of them coincides with the Zariski closure of the image of the given rational motion, which is irreducible by construction. This situation occurs in the example given on the right of Figure 6, since the variety of relative positions between base and end effector has two components (see Lemma 6.6. 

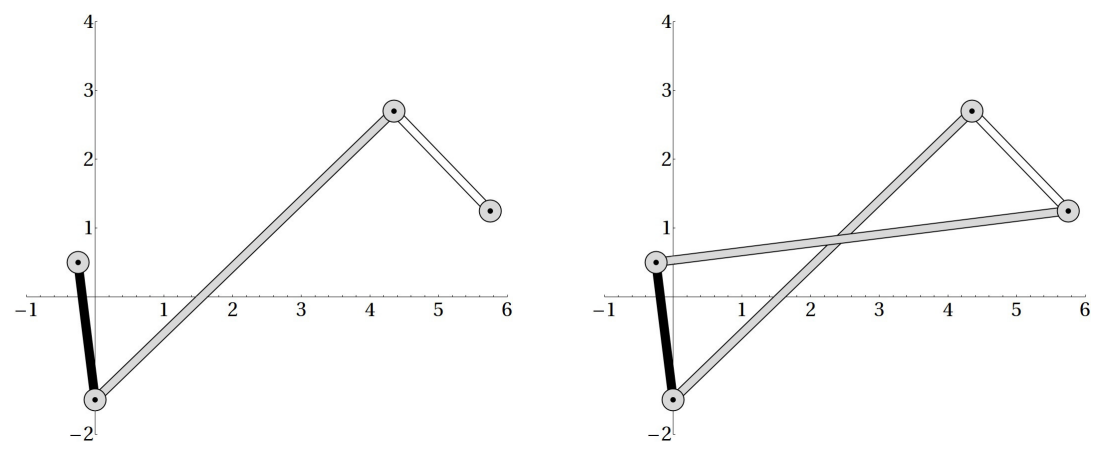

Figure 6 . Two linkages that both realize the motion given by $(t+\imath-3 \eta) \cdot(t+2 \imath+\eta(18+3 \imath))$, discussed in Example 4.4 . The depicted position is reached at $t=-2$. The linkage on the left has two degrees of freedom and thus weakly realizes the given motion as the relative position between base (in black) and end effector (in white). The linkage on the right has mobility one and provides a strong realization of this motion.

\section{FACTORIZATION OF MOTION POLYNOMials}

Motivated by the considerations from the previous section, we now address the problem of factorizing a motion polynomial $P=Z+\eta W \in \mathbb{K}[t]$ into linear factors. The main results are Theorem 5.15 and the corresponding algorithm FactorMotionPolynomial. To achieve them, we have to go through some technicalities, which however will not be needed in the rest of the paper.

We restrict our attention to monic polynomials, i.e., to polynomials $P$ whose leading coefficient is 1 , which implies that $\operatorname{deg} W<\operatorname{deg} Z$. Our goal is to write a monic $P \in \mathbb{K}[t]$ in the form $P=P_{1} \cdots P_{n}$, where $n=\operatorname{deg} P$ and $\operatorname{deg} P_{i}=1$ for all $i$. If $P=Z+\eta W$ and $P_{i}=Z_{i}+\eta W_{i}$, then $Z=Z_{1} \cdots Z_{n}$. Hence the primal part of each $P_{i}$ has to be one of the linear factors of $Z \in \mathbb{C}[t]$. We start by a few definitions and results characterizing monic motion polynomials that split into linear factors.

Definition 5.1. Let $\mathbf{z}=\left(z_{1}, \ldots, z_{n}\right) \in \mathbb{C}^{n}$. We define the following polynomials:

$$
Q_{i}(\mathbf{z}):=\left(t-\overline{z_{1}}\right) \cdots\left(t-\overline{z_{i-1}}\right)\left(t-z_{i+1}\right) \cdots\left(t-z_{n}\right) \text { for } i \in\{1, \ldots, n\} .
$$

Hence we have that $\operatorname{deg} Q_{i}(\mathbf{z})=n-1$ for all $i$.

Remark 5.2. Let $Z \in \mathbb{C}[t]$ and let $\mathbf{z}=\left(z_{1}, \ldots, z_{n}\right)$ be a fixed permutation of the roots of $Z$. Then the polynomials $Q_{i}(\mathbf{z})$ have a non-trivial ged if and only if the polynomial $Z$ admits a pair of complex-conjugate roots $\alpha$ and $\bar{\alpha}$ (this includes the case of multiple real roots).

Lemma 5.3. Let $P=Z+\eta W \in \mathbb{K}[t]$ be a monic motion polynomial, and let $\mathbf{z}=\left(z_{1}, \ldots, z_{n}\right)$ be a fixed permutation of the roots of $Z$ over $\mathbb{C}$. Then $P$ admits a factorization $P=P_{1} \cdots P_{n}$, where $P_{i}(t)=\left(t-z_{i}\right)+\eta w_{i}$ with $w_{i} \in \mathbb{C}$, if and only if $W$ lies in $\left\langle Q_{1}(\mathbf{z}), \ldots, Q_{n}(\mathbf{z})\right\rangle_{\mathbb{C}}$, i.e., in the $\mathbb{C}$-linear span of $Q_{1}(\mathbf{z}), \ldots, Q_{n}(\mathbf{z})$. 
Proof. Suppose that $P \in \mathbb{K}[t]$ admits a factorization into linear factors:

$$
P(t)=\prod_{i=1}^{n}\left(t-z_{i}+\eta w_{i}\right)=\prod_{i=1}^{n}\left(t-z_{i}\right)+\eta \sum_{k=1}^{n} \underbrace{\left(\prod_{j=1}^{k-1}\left(t-\overline{z_{j}}\right)\right)\left(\prod_{j=k+1}^{n}\left(t-z_{j}\right)\right)}_{=Q_{k}(\mathbf{z})} w_{k} .
$$

Such a factorization exists if and only if we can choose $w_{1}, \ldots, w_{n} \in \mathbb{C}$ such that $\sum_{k=1}^{n} w_{k} Q_{k}(\mathbf{z})$ matches the prescribed $\eta$-part of $P(t)=Z(t)+\eta W(t)$, namely if and only if $W \in\left\langle Q_{1}(\mathbf{z}), \ldots, Q_{n}(\mathbf{z})\right\rangle_{\mathbb{C}}$.

Lemma 5.4. Let $P \in \mathbb{K}[t]$ be a monic motion polynomial and suppose that $Z=$ $\operatorname{pp}(P)$ has no pair of complex-conjugate roots. Then for every permutation $\mathbf{z}=$ $\left(z_{1}, \ldots, z_{n}\right)$ of the roots of $Z$, the polynomial $P$ admits a factorization $P=P_{1} \cdots P_{n}$ with $\operatorname{pp}\left(P_{i}\right)=t-z_{i}$ for all $i$.

Proof. We fix a permutation $\mathbf{z}$ of the roots of $Z$, and we denote $Q_{k}=Q_{k}(\mathbf{z})$ for all $k$. From Lemma 5.3, we know that $P=Z+\eta W$ admits a factorization if and only if $W \in\left\langle Q_{1}, \ldots, Q_{n}\right\rangle_{\mathbb{C}}$. Clearly, this is always possible (for arbitrary $W$ ) if the following matrix $M_{n} \in \mathbb{C}^{n \times n}$ is non-singular:

$$
M_{n}=\left(\begin{array}{ccc}
\left\langle t^{0}\right\rangle Q_{1} & \cdots & \left\langle t^{0}\right\rangle Q_{n} \\
\left\langle t^{1}\right\rangle Q_{1} & \cdots & \left\langle t^{1}\right\rangle Q_{n} \\
\vdots & & \vdots \\
\left\langle t^{n-1}\right\rangle Q_{1} & \cdots & \left\langle t^{n-1}\right\rangle Q_{n}
\end{array}\right)
$$

Here $\left\langle t^{i}\right\rangle Q_{k}$ denotes the coefficient of $t^{i}$ in the polynomial $Q_{k}$. Notice that the matrix entries are, up to sign, elementary symmetric polynomials in the $z_{i}$ and $\overline{z_{i}}$. We now exhibit that the determinant of $M_{n}$ is nonzero under the assumptions on the roots of $Z$. Indeed, we find that

$$
\operatorname{det}\left(M_{n}\right)=\prod_{1 \leq i<j \leq n}\left(\overline{z_{i}}-z_{j}\right),
$$

which follows, as a special case, from Lemma 3 in [18] (by substituting $A_{k}=-z_{k}$, $B_{k}=-\overline{z_{k-1}}$, and by extracting the coefficient of $\left.\prod_{i=1}^{n} X_{i}^{i-1}\right)$. A similar determinant evaluation is given in [19] where the $z_{i}$ appear without conjugation. Since $\operatorname{det}\left(M_{n}\right)$ is very much reminiscent of the Vandermonde determinant, it is no surprise that (5.1) can also be proved in an analogous fashion.

Corollary 5.5. Let $\mathbf{z}=\left(z_{1}, \ldots, z_{n}\right) \in \mathbb{C}^{n}$ such that $\operatorname{gcd}\left(Q_{1}(\mathbf{z}), \ldots, Q_{n}(\mathbf{z})\right)=1$. Then

$$
\left\langle Q_{1}(\mathbf{z}), \ldots, Q_{n}(\mathbf{z})\right\rangle_{\mathbb{C}}=\{W \in \mathbb{C}[t]: \operatorname{deg}(W)<n\} .
$$

Proof. The gcd condition implies that $\mathbf{z}$ has no pair of complex-conjugate roots, as noticed in Remark5.2. Then the claim follows from Lemma 5.3 and Lemma 5.4.

Since the gcd of the polynomials $Q_{i}(\mathbf{z})$ introduced in Definition 5.1 plays an important role for the factorization of motion polynomials, we make some effort to describe it precisely (Lemma 5.6. Corollary 5.7. Proposition 5.10); for this purpose the combinatorial notion of matching will be introduced. At the same time we aim at a nicer characterization of those secondary parts in Lemma 5.3 that ensure the existence of a factorization (Proposition 5.12). To simplify the proofs of Propositions 5.10 and 5.12 we formulate the somewhat technical Lemma 5.9 . 
Lemma 5.6. Let $\alpha, \beta \in \mathbb{C}$ with $\alpha \neq \beta \neq \bar{\alpha}$, and let $\mathbf{z}=\left(z_{1}, \ldots, z_{n}\right) \in \mathbb{C}^{n}$ and $\widetilde{\mathbf{z}}=$ $\left(z_{1}, \ldots, z_{j-1}, \beta, z_{j}, \ldots, z_{n}\right) \in \mathbb{C}^{n+1}$ for some $j \in\{1, \ldots, n+1\}$. Then the multiplicities of the roots $\alpha$ and $\bar{\alpha}$ in $\operatorname{gcd}\left(Q_{1}(\mathbf{z}), \ldots, Q_{n}(\mathbf{z})\right)$ and in $\operatorname{gcd}\left(Q_{1}(\widetilde{\mathbf{z}}), \ldots, Q_{n+1}(\widetilde{\mathbf{z}})\right)$ are the same.

Proof. First observe that $Q_{k}(\mathbf{z})$ and $Q_{\ell}(\widetilde{\mathbf{z}})$ have the same multiplicities of $\alpha$ and $\bar{\alpha}$, where $\ell=k$ if $k<j$ and $\ell=k+1$ if $k \geq j$. On the other hand, the polynomial $Q_{j}(\widetilde{\mathbf{z}})$ has no such counterpart among the $Q_{k}(\mathbf{z})$, but its multiplicities of $\alpha$ and $\bar{\alpha}$ are greater than or equal to those in its (one or two) neighbors $Q_{j-1}(\widetilde{\mathbf{z}})$ and $Q_{j+1}(\widetilde{\mathbf{z}})$. The claim follows.

Corollary 5.7. Let $\mathbf{x} \in \mathbb{C}^{m}$ and $\mathbf{y} \in \mathbb{C}^{n}$ with $x_{i} \neq y_{j} \neq \overline{x_{i}}$ for all $1 \leq i \leq m$ and $1 \leq j \leq n$. If $\mathbf{z} \in \mathbb{C}^{m+n}$ is an arbitrary interlacement of $\mathbf{x}$ and $\mathbf{y}$, i.e., there are $1 \leq i_{1} \leq \cdots \leq i_{m} \leq m+n$ and $1 \leq j_{1} \leq \cdots \leq j_{n} \leq m+n$ with $\left\{i_{1}, \ldots, i_{m}\right\} \cap\left\{j_{1}, \ldots, j_{n}\right\}=\emptyset$ such that $z_{i_{k}}=x_{k}, 1 \leq k \leq m$, and $z_{j_{k}}=y_{k}$, $1 \leq k \leq n$, then

$$
\operatorname{gcd}\left(Q_{1}(\mathbf{z}), \ldots, Q_{m+n}(\mathbf{z})\right)=\operatorname{gcd}\left(Q_{1}(\mathbf{x}), \ldots, Q_{m}(\mathbf{x})\right) \cdot \operatorname{gcd}\left(Q_{1}(\mathbf{y}), \ldots, Q_{n}(\mathbf{y})\right) .
$$

Proof. This is a direct consequence of Lemma 5.6 .

Definition 5.8. Let $\mathbf{z}=\left(z_{1}, \ldots, z_{n}\right) \in \mathbb{C}^{n}$. A set

$$
M \subseteq\left\{(i, j): 1 \leq i<j \leq n \text { and } z_{i}=\overline{z_{j}}\right\}
$$

is called a matching of $\mathbf{z}$ if for all $\left(i_{1}, j_{1}\right),\left(i_{2}, j_{2}\right) \in M$ we have $i_{1} \neq i_{2}$ and $j_{1} \neq j_{2}$. (Note that for each $\mathbf{z} \in \mathbb{C}^{n}$ there is a bipartite graph with at most $2 n-2$ vertices such that the matchings in that graph and the matchings of $\mathbf{z}$ are in bijection.)

Lemma 5.9. Let $\mathbf{z}=\left(z_{1}, \ldots, z_{n}\right) \in \mathbb{C}^{n}$, let $G=\operatorname{gcd}\left(Q_{1}(\mathbf{z}), \ldots, Q_{n}(\mathbf{z})\right)$, and let $g=\operatorname{deg} G$. For a matching $M$ of $\mathbf{z}$ define $H(M):=\prod_{(i, j) \in M}\left(t-z_{j}\right)$. Then the following statements hold:

(i) For any matching $M$ of $\mathbf{z}$ we have $H(M) \mid G$.

(ii) There is a matching $M$ of $\mathbf{z}$ such that $H(M)=G$.

(iii) There are indices $1 \leq i_{1} \leq \cdots \leq i_{n-g} \leq n$ such that, setting $\widetilde{\mathbf{z}}=$ $\left(z_{i_{1}}, \ldots, z_{i_{n-g}}\right)$, we have $Q_{k}(\widetilde{\mathbf{z}})=\bar{Q}_{i_{k}}(\mathbf{z}) / G$ for all $1 \leq k \leq n-g$ and $\operatorname{gcd}\left(Q_{1}(\widetilde{\mathbf{z}}), \ldots, Q_{n-g}(\widetilde{\mathbf{z}})\right)=1$.

Proof. For (i) we show that $H(M)$ divides $Q_{k}(\mathbf{z})$ for any matching $M$ of $\mathbf{z}$ and for any $k \in\{1, \ldots, n\}$. Indeed, by rewriting

$$
H(M)=\prod_{(i, j) \in M}\left(t-z_{j}\right)=\left(\prod_{\substack{(i, j) \in M \\ j>k}}\left(t-z_{j}\right)\right)\left(\prod_{\substack{(i, j) \in M \\ j \leq k}}\left(t-\overline{z_{i}}\right)\right)
$$

the claim follows, since $Q_{k}(\mathbf{z})$ has precisely the roots $\overline{z_{1}}, \ldots, \overline{z_{k-1}}, z_{k+1}, \ldots, z_{n}$.

For (ii) and (iii) note that the statements trivially hold for $G=1$, so we assume now that $G \neq 1$. Using Corollary 5.7 the proof is reduced to the case where $\mathbf{z} \in\{\alpha, \bar{\alpha}\}^{n}$ for some $\alpha \in \mathbb{C}$. If $\alpha \in \mathbb{R}$ then we have $Q_{1}(\mathbf{z})=\cdots=Q_{n}(\mathbf{z})=$ $(t-\alpha)^{n-1}=G$ and we can choose $M:=\{(1,2),(2,3), \ldots,(n-1, n)\}$ and $i_{k}=k$ for $1 \leq k<n$. Hence from now on we assume $\alpha \neq \bar{\alpha}$.

Both assertions (ii) and (iii) are proved in parallel by an inductive argument on the degree of $G$, so in the following we show how to reduce the given scenario to one with smaller $g$. The main difficulty here is to pick an index $j$ such that 
deleting $z_{j}$ from $\mathbf{z}$ yields a gcd of smaller degree. We distinguish three cases and assume w.l.o.g. that $z_{n}=\alpha$ (case A) resp. $z_{1}=\alpha$ (cases B and C).

Case A: There exists an index $j$ such that $\#_{\alpha}\left(z_{j}, \ldots, z_{n}\right) \leq \#_{\bar{\alpha}}\left(z_{j}, \ldots, z_{n}\right)$, where $\#_{\alpha}$ denotes the number of occurrences of $\alpha$. In this situation we set $\widetilde{\mathbf{z}}=$ $\left(z_{1}, \ldots, z_{n-1}\right)$ and $\widetilde{G}=\operatorname{gcd}\left(Q_{1}(\widetilde{\mathbf{z}}), \ldots, Q_{n-1}(\widetilde{\mathbf{z}})\right)$. By the induction hypothesis there exists a matching $\widetilde{M}$ for $\widetilde{\mathbf{z}}$ such that $H(\widetilde{M})=\widetilde{G}$. Because of the assumption on the number of $\alpha$ 's and $\bar{\alpha}$ 's, there must be an index $i \in\{j, \ldots, n-1\}$ with $z_{i}=\bar{\alpha}$ that does not occur as the first entry of any pair in $\widetilde{M}$. Hence $M:=\widetilde{M} \cup\{(i, n)\}$ is a matching for $\mathbf{z}$ and we have $H(M)=(t-\alpha) H(\widetilde{M})$. Since by (i) we have $H(M) \mid G$, we obtain $(t-\alpha) \widetilde{G} \mid G$. Next observe that $Q_{k}(\widetilde{\mathbf{z}})=Q_{k}(\mathbf{z}) /\left(t-z_{n}\right)$ for all $1 \leq k<n$ and hence

$$
\widetilde{G}=\operatorname{gcd}\left(Q_{1}(\widetilde{\mathbf{z}}), \ldots, Q_{n-1}(\widetilde{\mathbf{z}})\right)=\frac{1}{t-\alpha} \operatorname{gcd}\left(Q_{1}(\mathbf{z}), \ldots, Q_{n-1}(\mathbf{z})\right) .
$$

From the definition of $G$ it follows that $G \mid(t-\alpha) \widetilde{G}$, and thus $G=(t-\alpha) \widetilde{G}=H(M)$.

Case B: There exists an index $j$ such $\#_{\alpha}\left(z_{1}, \ldots, z_{j}\right) \leq \#_{\bar{\alpha}}\left(z_{1}, \ldots, z_{j}\right)$. The reasoning in this case is completely analogous to case $\mathbf{A}$, setting $\widetilde{\mathbf{z}}=\left(z_{2}, \ldots, z_{n}\right)$.

Case $C$ : Neither case A nor case B applies, which means that for all indices $j$ we have $\#_{\alpha}\left(z_{1}, \ldots, z_{j}\right)>\#_{\bar{\alpha}}\left(z_{1}, \ldots, z_{j}\right)$ and $\#_{\alpha}\left(z_{j}, \ldots, z_{n}\right)>\#_{\bar{\alpha}}\left(z_{j}, \ldots, z_{n}\right)$. Note that in this situation we automatically have $z_{1}=z_{n}$, which w.l.o.g. we assumed to be $\alpha$. Now there exists no entry in $\mathbf{z}$ whose removal would yield a gcd of degree $\operatorname{deg} G-1$. We circumvent this problem by setting $\widetilde{\mathbf{z}}=\left(z_{1}, \ldots, z_{j-1}, z_{j+2}, \ldots, z_{n}\right)$ where $j$ is the largest index such that $z_{j}=\bar{\alpha}$; in particular $j<n$. The rest is analogous to the previous reasoning. As before, we may assume that there exists a matching $\widetilde{M}$ of $\widetilde{\mathbf{z}}$ with $H(\widetilde{M})=\widetilde{G}=\operatorname{gcd}\left(Q_{1}(\widetilde{\mathbf{z}}), \ldots, Q_{n-2}(\widetilde{\mathbf{z}})\right)$. Since $\#_{\alpha}\left(z_{1}, \ldots, z_{j}\right)>\#_{\bar{\alpha}}\left(z_{1}, \ldots, z_{j}\right)$ there exists an index $i<j$ with $z_{i}=\alpha$ such that $i$ does not appear as the first entry of any pair in $\widetilde{M}$. In order to extend $\widetilde{M}$ to a matching for $\mathbf{z}$ we need some relabeling; for this purpose we define $\tau(a)=a$ if $a<j$ and $\tau(a)=a+2$ otherwise. Then

$$
M:=\{(\tau(a), \tau(b)) \mid(a, b) \in \widetilde{M}\} \cup\{(i, j),(j, j+1)\}
$$

is a matching for $\mathbf{z}$ and $H(M)=(t-\alpha)(t-\bar{\alpha}) H(\widetilde{M})$; hence $(t-\alpha)(t-\bar{\alpha}) \widetilde{G} \mid G$. A simple calculation shows that $Q_{k}(\widetilde{\mathbf{z}})=Q_{k}(\mathbf{z}) /((t-\alpha)(t-\bar{\alpha}))$ for $k<j$ and that $Q_{k}(\widetilde{\mathbf{z}})=Q_{k+2}(\mathbf{z}) /((t-\alpha)(t-\bar{\alpha}))$ for $k \geq j$. Thus we get

$$
\widetilde{G}=\frac{1}{(t-\alpha)(t-\bar{\alpha})} \operatorname{gcd}\left(Q_{1}(\mathbf{z}), \ldots, Q_{j-1}(\mathbf{z}), Q_{j+2}(\mathbf{z}), \ldots, Q_{n}(\mathbf{z})\right) .
$$

Again, this implies $G=(t-\alpha)(t-\bar{\alpha}) \widetilde{G}=H(M)$, which concludes the proof.

Proposition 5.10. Let $\mathbf{z}=\left(z_{1}, \ldots, z_{n}\right) \in \mathbb{C}^{n}$ and let $M$ be a matching of $\mathbf{z}$ of maximal size, and let $Q_{1}(\mathbf{z}), \ldots, Q_{n}(\mathbf{z})$ be as in Definition 5.1. Then we have

$$
G:=\operatorname{gcd}\left(Q_{1}(\mathbf{z}), \ldots, Q_{n}(\mathbf{z})\right)=\prod_{(i, j) \in M}\left(t-z_{j}\right)
$$

(where the gcd is assumed to be a monic polynomial).

Proof. In Lemma 5.9 part(i) it was shown that the product on the right-hand side, which we denoted by $H(M)$, divides $G$ for any matching $M$. For the other direction we have to argue that the maximality of $M$ implies that $G \mid H(M)$, or equivalently, 
that $G \nmid H(M)$ implies that $M$ is not maximal. From Lemma 5.9 part (ii) it follows that there exists a matching $M^{\prime}$ of $\mathbf{z}$ such that $H\left(M^{\prime}\right)=G$. Note that $\left|M^{\prime}\right|=\operatorname{deg}(G)$. Thus, if $G \nmid H(M)$, which means that $\operatorname{deg}(H(M))<\operatorname{deg}(G)$, then $M$ is not maximal because $|M|<\left|M^{\prime}\right|$.

Example 5.11. Let $Z=(t-\alpha)^{r}(t-\bar{\alpha})^{r+1}$ for some $\alpha \in \mathbb{C} \backslash \mathbb{R}$. In the following table we consider different permutations $\mathbf{z} \in\{\alpha, \bar{\alpha}\}^{2 r+1}$ of the roots of $Z$; to each permutation we give the $\operatorname{gcd} G=\operatorname{gcd}\left(Q_{1}(\mathbf{z}), \ldots, Q_{n}(\mathbf{z})\right)$ and a matching $M$ of maximal size that witnesses $G$ :

\begin{tabular}{ccc}
\hline permutation & $G$ & $M$ \\
\hline$(\alpha, \ldots, \alpha, \bar{\alpha}, \ldots, \bar{\alpha})$ & $(t-\bar{\alpha})^{r}$ & $\{(1, r+1),(2, r+2), \ldots,(r, 2 r)\}$ \\
$(\bar{\alpha}, \ldots, \bar{\alpha}, \alpha, \ldots, \alpha)$ & $(t-\alpha)^{r}$ & $\{(1, r+2),(2, r+3), \ldots,(r, 2 r+1)\}$ \\
$(\bar{\alpha}, \alpha, \bar{\alpha}, \alpha, \ldots, \alpha, \bar{\alpha})$ & $(t-\alpha)^{r}(t-\bar{\alpha})^{r}$ & $\{(1,2),(2,3), \ldots,(2 r, 2 r+1)\}$
\end{tabular}

The cases displayed above are the extreme ones: indeed, it is easy to see that $r \leq \operatorname{deg}(G) \leq 2 r$. Moreover, for any $G=(t-\alpha)^{i}(t-\bar{\alpha})^{j}$ with $0 \leq i, j \leq r$ and $i+j \geq r$ there exists a permutation $\mathbf{z}$ that produces exactly this gcd $G$. These considerations lead to interesting combinatorial questions - e.g., how many permutations of a given set of roots are there that produce a prescribed gcd which, however, are irrelevant for our purposes.

Proposition 5.12. Let $\mathbf{z}=\left(z_{1}, \ldots, z_{n}\right) \in \mathbb{C}^{n}$ and $W \in \mathbb{C}[t]$ with $\operatorname{deg} W<n$. Then

$$
W \in\left\langle Q_{1}(\mathbf{z}), \ldots, Q_{n}(\mathbf{z})\right\rangle_{\mathbb{C}} \Longleftrightarrow W \in\left(Q_{1}(\mathbf{z}), \ldots, Q_{n}(\mathbf{z})\right) \cdot \mathbb{C}[t]
$$

where $\left(Q_{1}(\mathbf{z}), \ldots, Q_{n}(\mathbf{z})\right) \cdot \mathbb{C}[t]$ is the ideal of $\mathbb{C}[t]$ generated by $Q_{1}(\mathbf{z}), \ldots, Q_{n}(\mathbf{z})$.

Proof. The claim is equivalent to the following statement:

$$
\left\langle Q_{1}(\mathbf{z}), \ldots, Q_{n}(\mathbf{z})\right\rangle_{\mathbb{C}}=\left(Q_{1}(\mathbf{z}), \ldots, Q_{n}(\mathbf{z})\right) \cdot \mathbb{C}[t] \cap \mathbb{C}[t]_{<n},
$$

where $\mathbb{C}[t]_{<n}$ denotes the set of complex polynomials of degree less than $n$. Note that the containment " $\subseteq$ " is trivial. Let $G=\operatorname{gcd}\left(Q_{1}(\mathbf{z}), \ldots, Q_{n}(\mathbf{z})\right)$. If $G=1$, then by Corollary 5.5 we have $\left\langle Q_{1}(\mathbf{z}), \ldots, Q_{n}(\mathbf{z})\right\rangle_{\mathbb{C}}=\mathbb{C}[t]_{<n}$, which implies Equation (5.2). On the other hand, if $G$ is non-trivial, Equation 5.2 is equivalent to

$$
\left\langle\widetilde{Q}_{1}, \ldots, \widetilde{Q}_{n}\right\rangle_{\mathbb{C}}=\left(\widetilde{Q}_{1}, \ldots, \widetilde{Q}_{n}\right) \cdot \mathbb{C}[t] \cap \mathbb{C}[t]_{<n-g},
$$

where $\widetilde{Q}_{i}=Q_{i}(\mathbf{z}) / G$ and $g=\operatorname{deg} G$. By Lemma 5.9 part (iii), there exists $\widetilde{\mathbf{z}}=$ $\left(z_{i_{1}}, \ldots, z_{i_{n-g}}\right)$ such that $\operatorname{gcd}\left(Q_{1}(\widetilde{\mathbf{z}}), \ldots, Q_{n-g}(\widetilde{\mathbf{z}})\right)=1$, and $Q_{k}(\widetilde{\mathbf{z}})=\widetilde{Q}_{i_{k}}$ for all $k$. Hence, by Corollary 5.5 we have $\left\langle Q_{1}(\widetilde{\mathbf{z}}), \ldots, Q_{n-g}(\widetilde{\mathbf{z}})\right\rangle_{\mathbb{C}}=\mathbb{C}[t]_{<n-g}$. This implies Equation $[5.3)$, since $\left\langle Q_{1}(\widetilde{\mathbf{z}}), \ldots, Q_{n-g}(\widetilde{\mathbf{z}})\right\rangle_{\mathbb{C}} \subseteq\left\langle\widetilde{Q}_{1}, \ldots, \widetilde{Q}_{n}\right\rangle_{\mathbb{C}}$.

Recall that our goal is to decompose a given motion into a sequence of revolutions, by factorizing a corresponding motion polynomial. For this purpose we have to restrict the domain of motion polynomials we are working with: for example, there is no hope to write a motion whose orbits are unbounded as the composition of revolutions, since the orbits of revolutions are always bounded. In particular, if we consider a rational bounded curve, then it can be given by a parametrization $\varphi=(f / h, g / h)$ for some real polynomials $f, g$ and $h$ with $\operatorname{deg} h \geq \max \{\operatorname{deg} f, \operatorname{deg} g\}$. The boundedness of the curve implies that $h$ has no real roots. The limit of $\varphi(t)$ for $t \rightarrow \infty$ gives a point in $\mathbb{R}^{2}$, which can be translated to the origin. Then we even 
have $\operatorname{deg} h>\max \{\operatorname{deg} f, \operatorname{deg} g\}$. If we further assume that $h$ is monic, then also the motion polynomial $P=h+\eta(f+\imath g)$ is monic, and by Proposition 4.7 it traces the image of $\varphi$. This motivates the following definition.

Definition 5.13. Let $P=Z+\eta W$ be a motion polynomial in $\mathbb{K}[t]$. We say that $P$ is bounded if it is monic and if $Z$ does not have real roots. Notice that the motion $\phi$ given by a bounded motion polynomial is a map defined for all $t \in \mathbb{R}$; hence for such a motion we can use the notation $\phi: \mathbb{R} \longrightarrow \mathbb{P}_{\mathbb{R}}^{3}$ instead of $\phi: \mathbb{R} \rightarrow \mathbb{P}_{\mathbb{R}}^{3}$.

Remark 5.14. A bounded linear motion polynomial $P$ represents a revolution around a point: if $P(t)=t-k$, then $t-k \in \mathbb{K}$ represents a rotation around a point $Q$ that depends only on $k$. This follows from Lemma 4.3

We are now ready to state and prove the main result of this section:

Theorem 5.15. Let $P \in \mathbb{K}[t]$ be a bounded motion polynomial. Then there exists a real polynomial $R \in \mathbb{R}[t]$ such that $R P$ can be factored into linear polynomials.

If $P=Z+\eta W$ and $\widetilde{R}=\operatorname{gcd}(Z, \bar{Z})$, then the smallest such real polynomial is $R=\widetilde{R} / \operatorname{gcd}(\widetilde{R}, W \bar{W})$.

Proof. If $P$ is of the form $P=S \widetilde{P}$ with $S \in \mathbb{R}[t] \backslash \mathbb{R}$ and $\widetilde{P} \in \mathbb{K}[t]$, then we can apply the theorem to $\widetilde{P}$ obtaining $R \in \mathbb{R}[t]$ such that $R \widetilde{P}$ factors; thus also $R S \widetilde{P}=R P$ factors. Hence we may assume that $P$ does not have non-constant real factors.

Using Lemma 5.3 and Proposition 5.12, the proof is reduced to showing that there exists a permutation of the $n$ roots of $R Z$ (counted with multiplicities) such that the corresponding $G=\operatorname{gcd}\left(Q_{1}, \ldots, Q_{n}\right)$ divides $R W$. To this end write

$$
Z=\prod_{i=1}^{h}\left(\left(t-\alpha_{i}\right)^{r_{i}}\left(t-\overline{\alpha_{i}}\right)^{s_{i}}\right), \quad r_{i} \geq s_{i} \geq 0, \quad r_{i}>0,
$$

where $\alpha_{i} \in \mathbb{C}$ are pairwise conjugate-distinct, i.e., $\alpha_{i} \neq \alpha_{j}$ and $\alpha_{i} \neq \overline{\alpha_{j}}$ for $i \neq j$. Note that in the special case $s_{1}=\cdots=s_{h}=0$ we get $R=1$, in agreement with the fact that in this case already $P$ itself factors by Lemma 5.4. On the other hand, we have always $\operatorname{deg}(R) \leq \operatorname{deg}(P)$, and equality is attained when $r_{i}=s_{i}$ for all $i$ and $\operatorname{gcd}(Z, W)=1$.

Define $R_{i}:=\left(t-\alpha_{i}\right)\left(t-\overline{\alpha_{i}}\right)$ and write $\widetilde{R}=\prod_{i=1}^{h} R_{i}^{s_{i}}$. Next let $u_{i}$ and $v_{i}$ be the multiplicities of $\alpha_{i}$ and $\overline{\alpha_{i}}$ in $W$, i.e.,

$$
W=\widetilde{W} \cdot \prod_{i=1}^{h}\left(\left(t-\alpha_{i}\right)^{u_{i}}\left(t-\overline{\alpha_{i}}\right)^{v_{i}}\right)
$$

for some polynomial $\widetilde{W} \in \mathbb{C}[t]$ such that $\operatorname{gcd}\left(\widetilde{W}, R_{1} \cdots R_{h}\right)=1$. By introducing the quantity $m_{i}:=\min \left\{s_{i}, u_{i}+v_{i}\right\}$, the polynomial $R$ can be written as

$$
R=\frac{\widetilde{R}}{\operatorname{gcd}(\widetilde{R}, W \bar{W})}=\prod_{i=1}^{h} R_{i}^{s_{i}-m_{i}}
$$


and hence

$$
\begin{aligned}
R Z & =\prod_{i=1}^{h}\left(\left(t-\alpha_{i}\right)^{r_{i}+s_{i}-m_{i}}\left(t-\overline{\alpha_{i}}\right)^{2 s_{i}-m_{i}}\right), \\
R W & =\widetilde{W} \cdot \prod_{i=1}^{h}\left(\left(t-\alpha_{i}\right)^{u_{i}+s_{i}-m_{i}}\left(t-\overline{\alpha_{i}}\right)^{v_{i}+s_{i}-m_{i}}\right) .
\end{aligned}
$$

To construct an admissible permutation, the roots $\alpha_{i}, \overline{\alpha_{i}}$ are arranged as follows:

$$
(\underbrace{\overline{\alpha_{i}}, \ldots, \overline{\alpha_{i}}}_{s_{i}-\min \left\{s_{i}, v_{i}\right\}}, \underbrace{\alpha_{i}, \ldots, \alpha_{i}}_{r_{i}+s_{i}-m_{i}}, \underbrace{\overline{\alpha_{i}}, \ldots, \overline{\alpha_{i}}}_{s_{i}-\min \left\{s_{i}, u_{i}\right\}}) .
$$

The assumption on $P$ ensures that if $s_{i}>0$ then $u_{i}=0$ or $v_{i}=0$; indeed, if $s_{i}, u_{i}, v_{i}$ were all strictly positive, then $R_{i}$ would be a common real factor of $Z$ and $W$. Then $m_{i}=\min \left\{s_{i}, u_{i}\right\}+\min \left\{s_{i}, v_{i}\right\}$, and hence the previous multiplicities agree with those in $R Z$.

The final permutation is obtained as an arbitrary interlacement of all arrangements for $1 \leq i \leq h$. Proposition 5.10 then implies that

$$
G=\prod_{i=1}^{h}\left(\left(t-\alpha_{i}\right)^{s_{i}-\min \left\{s_{i}, v_{i}\right\}}\left(t-\overline{\alpha_{i}}\right)^{s_{i}-\min \left\{s_{i}, u_{i}\right\}}\right) .
$$

Since $s_{i}-\min \left\{s_{i}, v_{i}\right\} \leq u_{i}+s_{i}-m_{i}$ and $s_{i}-\min \left\{s_{i}, u_{i}\right\} \leq v_{i}+s_{i}-m_{i}$, we conclude that $G$ divides $R W$.

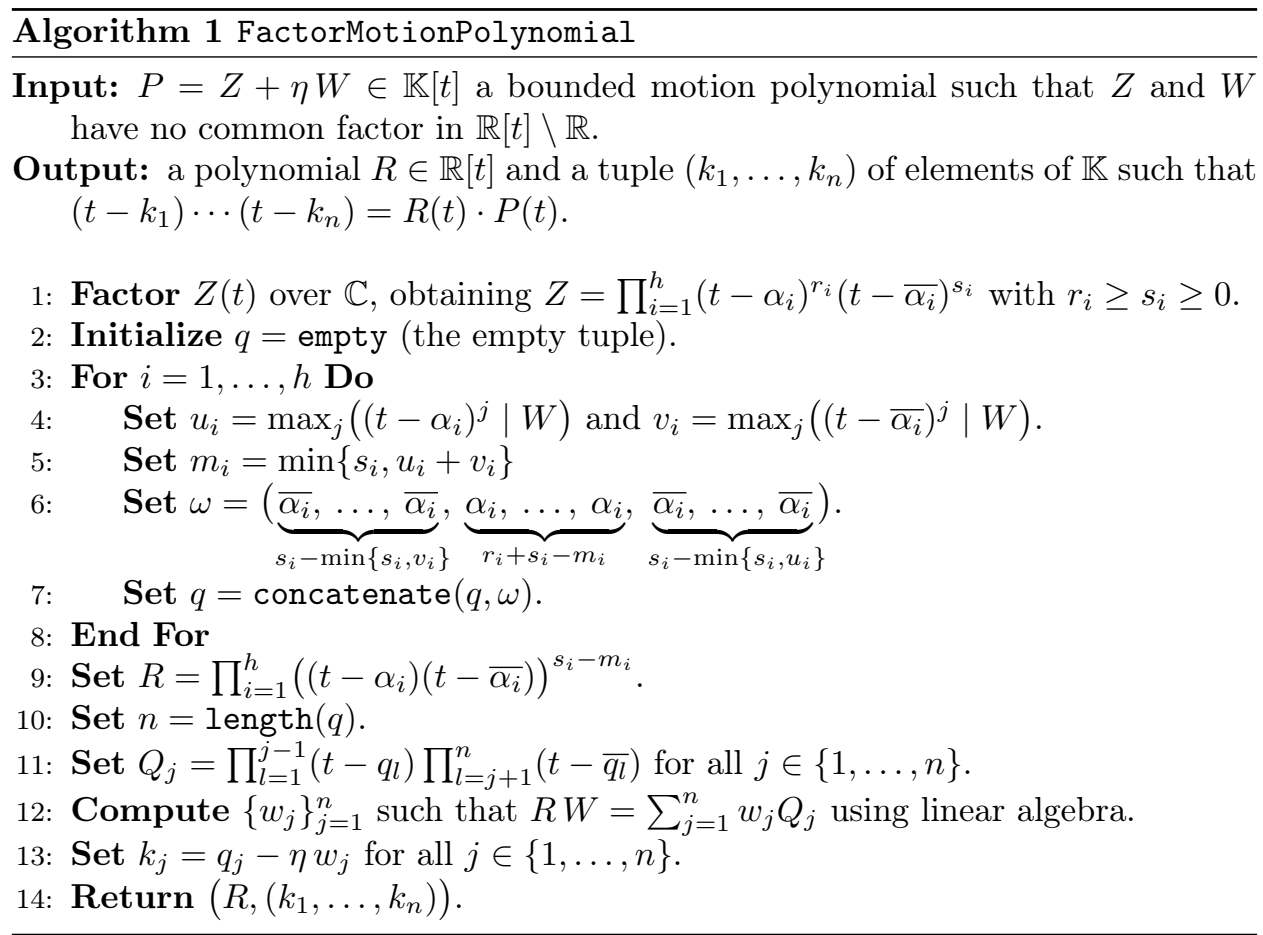

The proof of Theorem 5.15 immediately gives rise to a factorization algorithm for motion polynomials, called FactorMotionPolynomial. It produces one single 
factorization for a given polynomial $P$, although there could be many for the following reasons: first, there may be several admissible permutations of the roots of $R Z$, and second, the choice of the coefficients $\left\{w_{j}\right\}$ in Step 12 may not be unique, since the polynomials $\left\{Q_{j}\right\}$ need not be $\mathbb{C}$-linearly independent.

Example 5.16. We can review Example 4.12 in the light of Theorem 5.15 and the algorithm FactorMotionPolynomial. We consider a particular instance of the collection of motion polynomials

$$
P(t)=\left(t^{2}+1\right)+\eta(a t-b \imath)
$$

namely we choose $a=\imath$ and $b=2 \imath$, obtaining the motion we presented in Section 2 . If we run FactorMotionPolynomial with $P$ as input, we obtain $R=t^{2}+1$, and if we fix the permutation $(\imath,-\imath,-\imath, \imath)$ of the roots of $\mathrm{pp}(R P)$, as in 4.6), we obtain a two-dimensional family of factorizations into linear factors, i.e., the solutions of the linear system (4.7), out of which we choose the factorization used in Section 2 .

\section{THE FLIP PROCEDURE}

The procedure described in this section is crucial for the construction of linkages with mobility one. It is inspired by similar techniques - involving the interchange of factors in a factorization of a quadratic motion polynomial — used in [9] and [10].

Lemma 6.1. Let $k_{1}, k_{2} \in \mathbb{K}$ be such that $\mathrm{pp}\left(k_{1}\right) \neq \overline{\mathrm{pp}\left(k_{2}\right)}$. Then there exists a unique pair $\left(k_{3}, k_{4}\right) \in \mathbb{K}^{2}$ such that:

(i) $\mathrm{pp}\left(k_{3}\right)=\mathrm{pp}\left(k_{2}\right)$ and $\mathrm{pp}\left(k_{4}\right)=\mathrm{pp}\left(k_{1}\right)$;

(ii) $\left(t-k_{1}\right)\left(t-k_{2}\right)=\left(t-k_{3}\right)\left(t-k_{4}\right)$ as polynomials in $\mathbb{K}[t]$.

Proof. Let us suppose that $k_{i}=z_{i}+\eta w_{i}$ for $i \in\{1,2\}$. We make the ansatz $k_{3}=z_{2}+\eta w_{3}$ and $k_{4}=z_{1}+\eta w_{4}$, where $w_{3}$ and $w_{4}$ are elements of $\mathbb{C}$ to be determined. Then condition (ii) is equivalent to the linear system

$$
\left(\begin{array}{cc}
1 & 1 \\
z_{1} & \bar{z}_{2}
\end{array}\right)\left(\begin{array}{c}
w_{3} \\
w_{4}
\end{array}\right)=\left(\begin{array}{c}
w_{1}+w_{2} \\
\bar{z}_{1} w_{2}+z_{2} w_{1}
\end{array}\right)
$$

which has a unique solution, since by hypothesis the determinant of the matrix is different from zero.

Definition 6.2. Let $k_{1}, k_{2} \in \mathbb{K}$ be such that $\operatorname{pp}\left(k_{1}\right) \neq \overline{\operatorname{pp}\left(k_{2}\right)}$. Then we define $\operatorname{Flip}\left(k_{1}, k_{2}\right):=\left(k_{3}, k_{4}\right)$, where $\left(k_{3}, k_{4}\right) \in \mathbb{K}^{2}$ is the pair from Lemma 6.1.

Definition 6.3. Let $k=z+\eta w \in \mathbb{K}$ with $z \in \mathbb{C} \backslash \mathbb{R}$. Then $k$ represents a rotation around a point that we denote by $\chi(k)$. Using Equation 4.5 one obtains that

$$
\chi(k)=\frac{w}{\bar{z}-z} .
$$

Definition 6.4. Let $k_{1}, k_{2} \in \mathbb{K}$ be such that $\mathrm{pp}\left(k_{1}\right) \neq \overline{\mathrm{pp}\left(k_{2}\right)}$ and $\mathrm{pp}\left(k_{1}\right), \mathrm{pp}\left(k_{2}\right) \in$ $\mathbb{C} \backslash \mathbb{R}$. We define the flip linkage of $k_{1}, k_{2}$ via the algorithm FlipLinkage below. The graph of this flip linkage is shown in Figure 4.

Lemma 6.5. Let $k_{1}, k_{2} \in \mathbb{K}$ with $\overline{\operatorname{pp}\left(k_{1}\right)} \neq \operatorname{pp}\left(k_{2}\right)$. Let $\left(k_{3}, k_{4}\right)=\operatorname{Flip}\left(k_{1}, k_{2}\right)$. Then

$$
\left|\chi\left(k_{1}\right)-\chi\left(k_{2}\right)\right|=\left|\chi\left(k_{3}\right)-\chi\left(k_{4}\right)\right| .
$$

If, in addition, $z_{1}, z_{2} \notin \mathbb{R}$ and $\chi\left(k_{1}\right) \neq \chi\left(k_{2}\right)$, then the following holds:

$$
\left|\chi\left(k_{1}\right)-\chi\left(k_{2}\right)\right| \neq\left|\chi\left(k_{1}\right)-\chi\left(k_{3}\right)\right| \text {. }
$$




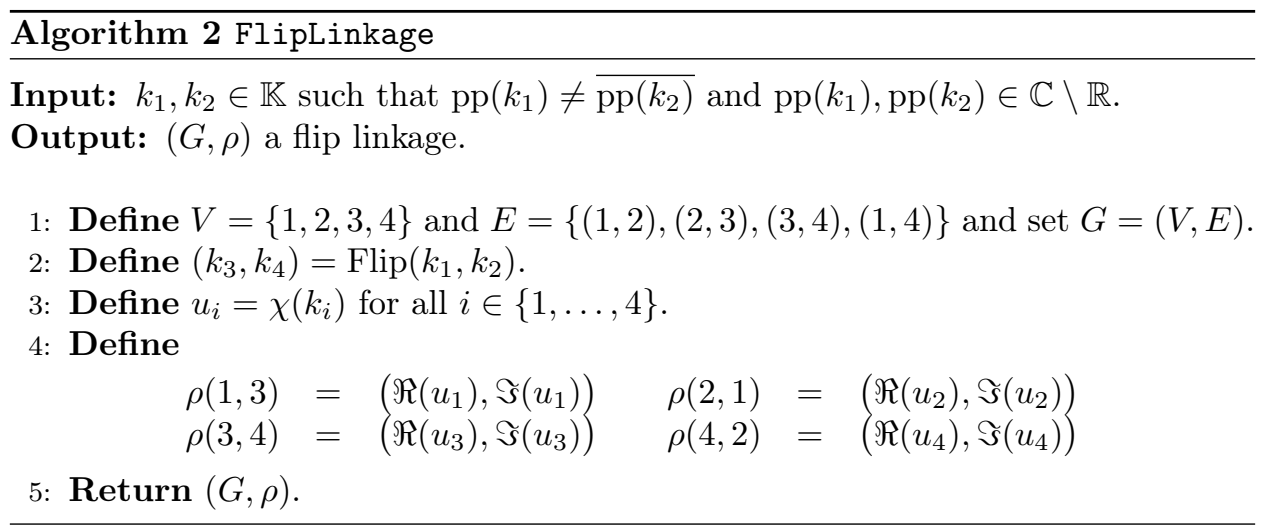

Proof. Let $k_{1}=z_{1}+\eta w_{1}$ and $k_{2}=z_{2}+\eta w_{2}$. Then the condition $\left(t-k_{1}\right)\left(t-k_{2}\right)=$ $\left(t-k_{3}\right)\left(t-k_{4}\right)$ implies that $k_{3}=z_{2}+\eta w_{3}$ and $k_{4}=z_{1}+\eta w_{4}$ with

$$
w_{3}=\frac{\left(z_{2}-\overline{z_{2}}\right) w_{1}+\left(\overline{z_{1}}-\overline{z_{2}}\right) w_{2}}{z_{1}-\overline{z_{2}}} \quad \text { and } \quad w_{4}=\frac{\left(z_{1}-z_{2}\right) w_{1}+\left(z_{1}-\overline{z_{1}}\right) w_{2}}{z_{1}-\overline{z_{2}}} .
$$

A direct calculation shows that

$$
\left|\chi\left(k_{3}\right)-\chi\left(k_{4}\right)\right|=\left|\frac{w_{3}}{\overline{z_{2}}-z_{2}}-\frac{w_{4}}{\overline{z_{1}}-z_{1}}\right|=\left|\frac{w_{1}}{\overline{z_{1}}-z_{1}}-\frac{w_{2}}{\overline{z_{2}}-z_{2}}\right|=\left|\chi\left(k_{1}\right)-\chi\left(k_{2}\right)\right|
$$

as claimed. The second claim is also obtained via a direct computation.

Lemma 6.5 implies that the linkages produced by the algorithm FlipLinkage have the shape of a (possibly degenerated) antiparallelogram, as in the right part of Figure 6. We introduce the notion of flip mobility to exclude some degenerated cases, namely the ones where the antiparallelogram is in fact a square.

Notation. Let $k_{1}=z_{1}+\eta w_{1}$ and $k_{2}=z_{2}+\eta w_{2}$ be two elements of $\mathbb{K}$. We say that the condition $\operatorname{FM}\left(k_{1}, k_{2}\right)$ (which stands for flip mobility) holds if and only if

$$
z_{1}, z_{2} \notin \mathbb{R}, \quad z_{1} \neq z_{2}, \quad z_{1} \neq \bar{z}_{2}, \quad \chi\left(k_{1}\right) \neq \chi\left(k_{2}\right) .
$$

Lemma 6.6. Let $k_{1}, k_{2} \in \mathbb{K}$ such that $\mathrm{FM}\left(k_{1}, k_{2}\right)$ holds. Then the linkage $L$ obtained by FlipLinkage $\left(k_{1}, k_{2}\right)$ has mobility one and the configuration curve has two components $C_{1}$ and $C_{2}$. Moreover, the natural maps $C_{1} \longrightarrow \operatorname{VRP}(i, j)$ and $C_{2} \longrightarrow \operatorname{VRP}(i, j)$ are isomorphisms for every two neighboring links $i$ and $j$.

Proof. Since the linkages obtained via FlipLinkage are constituted by four bars, this is a well-known result; see for example [4, Table on p. 426]. One can also prove the statement using the notions we introduced in Section 3 as follows. We set $L=$ FlipLinkage $\left(k_{1}, k_{2}\right)$, and denote $\left(k_{3}, k_{4}\right)=\operatorname{Flip}\left(k_{1}, k_{2}\right)$. Recalling Definition 3.6 . our goal is to prove that $\operatorname{Conf}(L)$ is a one-dimensional variety. One notices that

$$
\operatorname{Conf}(L) \cong\left\{\left(\lambda_{1}: \mu_{1}\right), \ldots,\left(\lambda_{4}: \mu_{4}\right): f_{12}\left(\lambda_{1}, \mu_{1}, \lambda_{2}, \mu_{2}\right)=f_{34}\left(\lambda_{3}, \mu_{3}, \lambda_{4}, \mu_{4}\right)\right\},
$$

where

$$
\begin{array}{cccc}
f_{12}: & \mathbb{P}_{\mathbb{R}}^{1} \times \mathbb{P}_{\mathbb{R}}^{1} & \longrightarrow & \mathbb{P}(\mathbb{K}) \\
& \left(\lambda_{1}: \mu_{1}\right),\left(\lambda_{1}: \mu_{1}\right) & \mapsto & \left(\lambda_{1}-\mu_{1} k_{1}\right)\left(\lambda_{2}-\mu_{2} k_{2}\right)
\end{array}
$$

and analogously for $f_{34}$. The images of $f_{12}$ and $f_{34}$ are two smooth quadrics whose intersection, equal to $\operatorname{Conf}(L)$, is a curve with two components. Using this it is possible to prove the claim. 
Remark 6.7. Results in Example 4.4 are particular instances of Lemma 6.6. One can in fact check that in that case $L=\mathrm{FlipLinkage}\left(k_{1}, k_{2}\right)$, where $k_{1}=\imath-\eta \cdot 3$ and $k_{2}=-2 \imath-\eta(2+\imath)$.

\section{Construction of Linkages}

In this section, we show how to construct a linkage with mobility one that traces an algebraic curve described by a rational parametrization. Based on the results of Section 5, we first describe an algorithm which takes a bounded motion polynomial $P$ and constructs a linkage weakly realizing $P$ (see Definition 4.14).

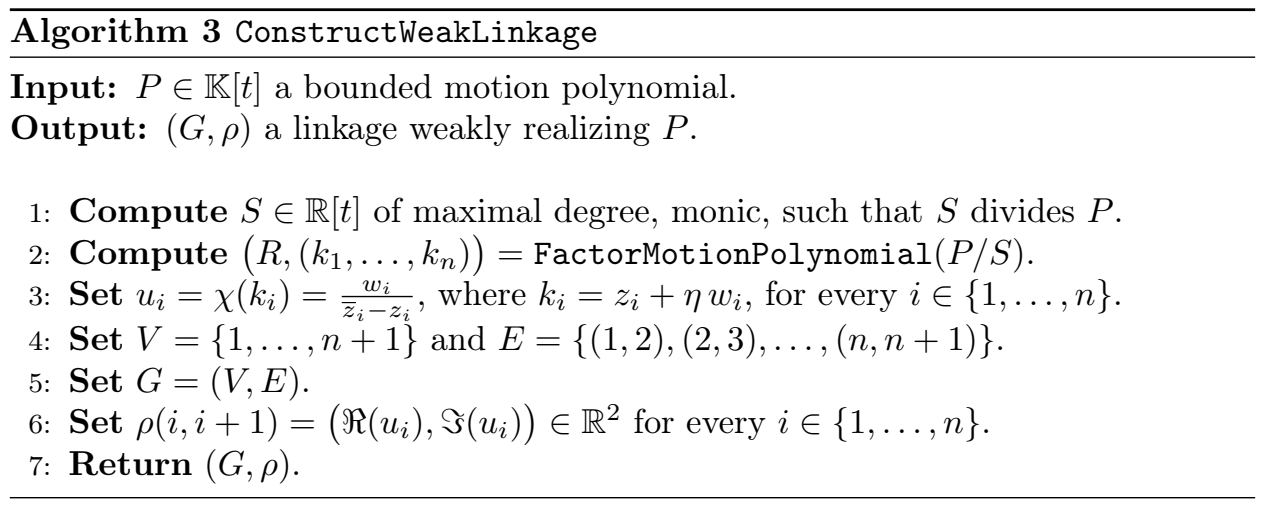

Proposition 7.1. Algorithm ConstructWeakLinkage is correct.

Proof. The linkage $L$ constructed by the algorithm ConstructWeakLinkage is an open chain, so its configuration space is isomorphic to $\left(\mathbb{P}_{\mathbb{C}}^{1}\right)^{n-1}$, where $n-1$ is the number of joints of $L$. Let $\phi: \mathbb{R} \longrightarrow \mathbb{P}_{\mathbb{C}}^{3}$ be the motion corresponding to $R P$. We consider links 1 and $n$, and we prove that image $(\phi) \subseteq \mathrm{RP}(1, n)$, namely that $L$ weakly realizes $R P$ according to Definition 4.14. In our situation we have that

$$
\operatorname{RP}(1, n)=\left\{\sigma_{1,2} \circ \sigma_{2,3} \circ \cdots \circ \sigma_{n-1, n}: \sigma_{i, i+1} \in \mathbb{P}_{\mathbb{C}}^{1}\right\} .
$$

Now we fix an arbitrary $t \in \mathbb{R}$ and we take $\sigma_{i, i+1}$ to be the isometry given by $t-k_{i}$ for all $i \in\{1, \ldots, n-1\}$. Then $\phi(t) \in \operatorname{RP}(1, n)$, so the claim is proven.

Suppose we are given a rational parametrization of a bounded curve. As we saw in Proposition 4.7, starting from it we can construct a bounded polynomial $P$ such that the curve is the orbit of the origin in $\mathbb{R}^{2}$ under the motion described by $P$. Then the algorithm ConstructWeakLinkage returns a linkage $L$ that weakly realizes $P$. However, this is rather unsatisfactory, since the configuration space of $L$ is, in general, a variety of high dimension. Our goal is to obtain a linkage with mobility one, providing a strong realization of $P$. The main idea is to "rigidify" $L$, which by construction is an open chain, by introducing additional links and joints, forming antiparallelograms. At the level of graphs, this corresponds to extending the linear graph of $L$ to a $2 \times n$ ladder graph (see Figure 7 ). To do so, we pick a linear motion polynomial $t-l$, which will constitute the first "step" of the ladder, and then apply the flip procedure iteratively to complete the ladder. In the following, we investigate how $l \in \mathbb{K}$ has to be chosen so that the resulting linkage has mobility one. 
Definition 7.2. Let $\mathbf{k}=\left(k_{1}, \ldots, k_{m}\right)$ be a tuple in $\mathbb{K}^{m}$, and let $l \in \mathbb{K}$. Define

$$
l_{1}:=l, \quad\left(\widetilde{k}_{i}, l_{i+1}\right):=\operatorname{Flip}\left(l_{i}, k_{i}\right) \quad \text { for all } i \in\{1, \ldots, m\} .
$$

Then we say that the condition $\operatorname{IFM}(l, \mathbf{k})$ (which stands for iterated flip mobility) holds if and only if $\operatorname{FM}\left(l_{i}, k_{i}\right)$ holds for all $i \in\{1, \ldots, m\}$.

In order to prove that the algorithm we propose works, we have to show that it is always possible to find an element $l \in \mathbb{K}$ such that the iterated flip mobility condition holds. For this we use a property of flip linkages.

Definition 7.3. Given an element $k=z+\eta w \in \mathbb{K}$ with $z \neq 0$, we $\operatorname{define} \operatorname{inv}(k)$ to be the element $\bar{z}-\eta w \in \mathbb{K}$. Recalling Remark 4.2 , we have that $k$ represents an isometry $\sigma$, and $\operatorname{inv}(k)$ its inverse $\sigma^{-1}$. Moreover, if $z \notin \mathbb{R}$, then $t-k$ represents an isometry for every $t \in \mathbb{R}$, and $t-\operatorname{inv}(k)$ the inverse isometry.

Lemma 7.4. Let $k_{1}, k_{2} \in \mathbb{K}$ be such that $\operatorname{pp}\left(k_{1}\right) \neq \overline{\operatorname{pp}\left(k_{2}\right)}$ and $\operatorname{pp}\left(k_{2}\right) \notin \mathbb{R}$. Let $\left(k_{3}, k_{4}\right)=\operatorname{Flip}\left(k_{1}, k_{2}\right)$. Then we have that

$$
\left(\operatorname{inv}\left(k_{3}\right), k_{1}\right)=\operatorname{Flip}\left(k_{4}, \operatorname{inv}\left(k_{2}\right)\right) .
$$

Proof. Notice that the fact that $\operatorname{pp}\left(k_{2}\right) \notin \mathbb{R}$ implies that the element $\left(t-k_{2}\right)$ represents an isometry for all $t \in \mathbb{R}$. From Definition 7.3 one has that the inverse isometry is represented by $\left(t-\operatorname{inv}\left(k_{2}\right)\right)$. The two situations are depicted below:
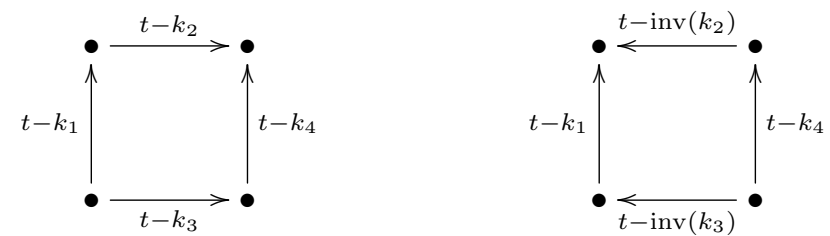

$$
\left(k_{3}, k_{4}\right)=\operatorname{Flip}\left(k_{1}, k_{2}\right) \quad\left(\operatorname{inv}\left(k_{3}\right), k_{1}\right)=\operatorname{Flip}\left(k_{4}, \operatorname{inv}\left(k_{2}\right)\right)
$$

A direct computation shows the desired result.

Lemma 7.5. Let $\mathbf{k}=\left(k_{1}, \ldots, k_{m}\right)$ be a tuple in $\mathbb{K}^{m}$ such that $\mathrm{pp}\left(k_{i}\right) \notin \mathbb{R}$ for all $i \in\{1, \ldots, m\}$. Then there exists $l \in \mathbb{K}$ with $\operatorname{pp}(l) \notin \mathbb{R}$ such that $\operatorname{IFM}(l, \mathbf{k})$ holds.

Proof. Following the notation of Definition 7.2 we have that $\operatorname{pp}\left(l_{i}\right)=\operatorname{pp}(l)$ for all $i \in\{1, \ldots, m\}$. Hence, in order to ensure that $\operatorname{IFM}(l, \mathbf{k})$ holds, we need to choose $l$ such that $\operatorname{pp}(l) \neq \operatorname{pp}\left(k_{i}\right)$ and $\operatorname{pp}(l) \neq \overline{\operatorname{pp}\left(k_{i}\right)}$ for all $i \in\{1, \ldots, m\}$. From now on, we fix such a value for $\mathrm{pp}(l)$. The other situations we should keep away from are the ones where $\chi\left(l_{i}\right)=\chi\left(k_{i}\right)$. Since $\operatorname{pp}\left(l_{i}\right)$ is now fixed, the condition $\chi\left(l_{i}\right)=\chi\left(k_{i}\right)$ becomes a linear equation for $\operatorname{sp}\left(l_{i}\right)$, which always admits a unique solution $\widehat{w_{i}} \in \mathbb{C}$. Let us define $\widehat{l_{i}}=\operatorname{pp}(l)+\eta \widehat{w_{i}}$. Using Lemma 7.4 repeatedly (namely $i-1$ times) starting from the pair $\left(\widehat{l_{i}}, \operatorname{inv}\left(k_{i-1}\right)\right)$, we obtain an element $h_{i} \in \mathbb{K}$ such that

$$
\chi\left(l_{i}\right) \neq \chi\left(k_{i}\right) \quad \Longleftrightarrow \quad l \neq h_{i} .
$$

Summing up, for every fixed value of $\operatorname{pp}(l)$ different from all $\left\{\operatorname{pp}\left(k_{i}\right)\right\}_{i=1}^{m}$ and all $\left\{\overline{\operatorname{pp}\left(k_{i}\right)}\right\}_{i=1}^{m}$, there are finitely many values for $\operatorname{sp}(l)$ that should be avoided for $\operatorname{IFM}(l, \mathbf{k})$ to hold. Thus we can always find $l \in \mathbb{K}$ with the claimed properties.

We are ready to present the algorithm ConstructStrongLinkage. Figure 7 shows the labeled graph produced by the algorithm. 


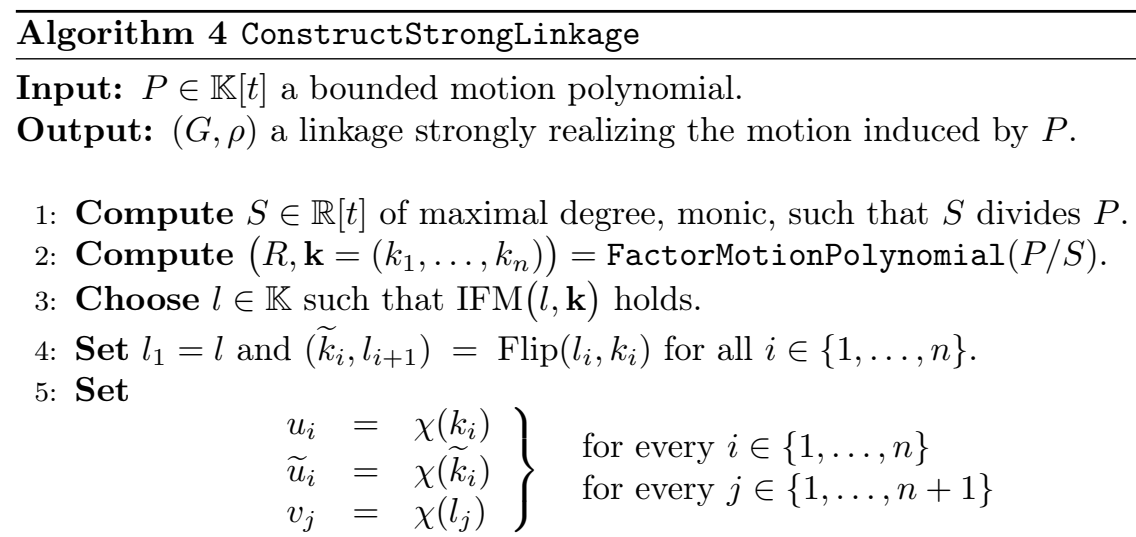

6: Set $V=\{1, \ldots, 2 n+2\}$.

7: Set $E=\{(i, i+1),(n+1+i, n+2+i),(j, n+1+j)$ for all $i \in\{1, \ldots, n\}$ and $j \in$ $\{1, \ldots, n+1\}\}$.

8: Set $G=(V, E)$.

9: Set

$$
\left.\begin{array}{rl}
\rho(i, i+1) & =\left(\Re\left(u_{i}\right), \Im\left(u_{i}\right)\right) \\
\rho(n+1+i, n+2+i) & =\left(\Re\left(\widetilde{u}_{i}\right), \Im\left(\widetilde{u}_{i}\right)\right) \\
\rho(j, n+1+j) & =\left(\Re\left(v_{j}\right), \Im\left(v_{j}\right)\right)
\end{array}\right\} \quad \begin{aligned}
& \text { for every } i \in\{1, \ldots, n\} \\
& \text { for every } j \in\{1, \ldots, n+1\}
\end{aligned}
$$

10: Return $(G, \rho)$.

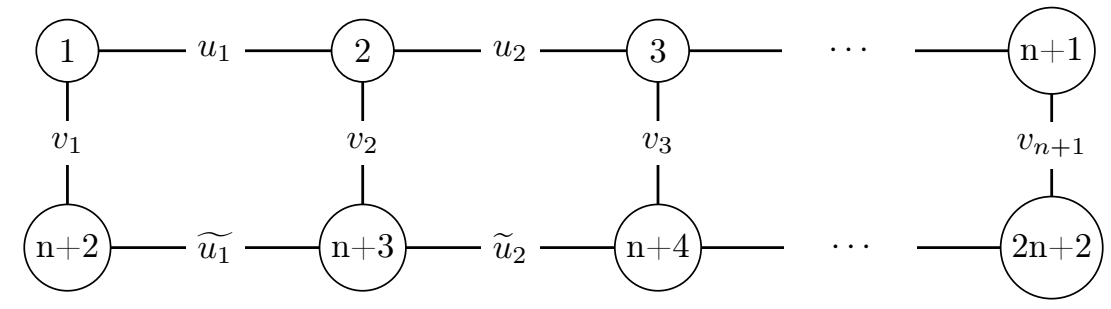

Figure 7. The labeled graph of the linkage returned by the algorithm ConstructStrongLinkage.

Theorem 7.6. Let $P$ be a bounded polynomial in $\mathbb{K}[t]$ and let $\phi: \mathbb{R} \longrightarrow \mathbb{P}_{\mathbb{R}}^{3}$ be the rational motion induced by $P$. Let us denote by $L$ the linkage obtained by applying ConstructStrongLinkage to $P$. Then $L$ strongly realizes the motion $\phi$.

Proof. Let $K$ be the configuration space of $L=(G, \rho)$. As in the statement of Lemma 6.6. we are going to prove that for any two neighboring edges $i$ and $j$ of $G$, every irreducible component of $K$ is isomorphic to $\operatorname{RP}(i, j)$. This will prove that $K$ has dimension one, which means that $L$ has mobility one.

Recall from Definition 3.4 that $K$ is the collection of configurations $\Sigma=\left(\sigma_{k, l}\right)$ satisfying the equations imposed by the directed cycles of $G$. First of all, notice 
that one can consider only those equations imposed by directed cycles of the form:

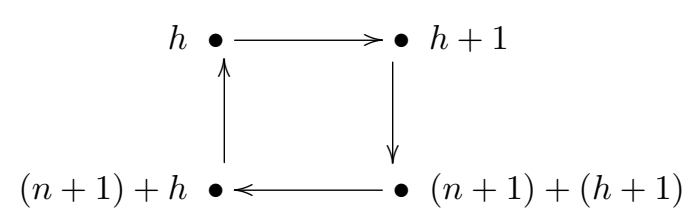

In fact, all other equations belong to the ideal generated by these ones. We introduce the following notation: for $h \in\{1, \ldots, n\}$, denote by $\operatorname{sq}(h)$ the set

$\{(h, h+1),(h+1,(n+1)+(h+1)),((n+1)+(h+1),(n+1)+h),((n+1)+h, h)\}$.

We define $\pi_{\mathrm{sq}(h)}$ to be the projection

$$
\pi_{\mathrm{sq}(h)}: K \longrightarrow \prod_{(r, s) \in \mathrm{sq}(h)} \operatorname{VRP}(r, s)
$$

Then the image of $\pi_{\mathrm{sq}(h)}$ is contained in the configuration space $C_{h}$ of a flip linkage, which by Lemma 6.6 has two components, so $C_{h}=C_{h}^{1} \cup C_{h}^{2}$.

For every sequence $b \in\{1,2\}^{n}$, we set

$$
K_{b}:=\pi_{\mathrm{sq}(1)}^{-1}\left(C_{1}^{b(1)}\right) \cap \cdots \cap \pi_{\mathrm{sq}(n)}^{-1}\left(C_{n}^{b(n)}\right) .
$$

By construction we have $K=\bigcup_{b \in\{1,2\}^{n}} K_{b}$. We are going to prove that for every sequence $b$ and for any two neighboring links $i$ and $j$ in $G$, we have $K_{b} \cong \operatorname{VRP}(i, j)$.

For the sake of simplicity, we start by proving this result when the graph $G$ consists only of two squares (i.e. $n=2$ ):

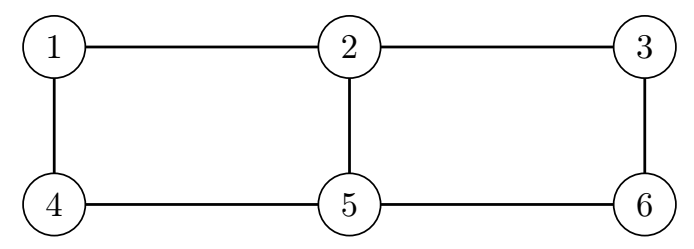

In this case we only have two flip linkages with configuration spaces $C_{1}$ and $C_{2}$. Let us fix $b \in\{1,2\}^{2}$, and consider the diagram:

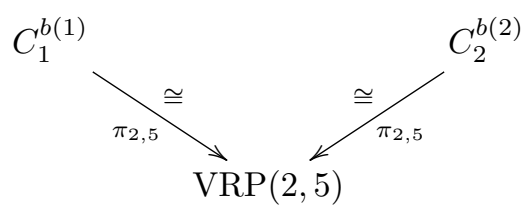

The maps $\pi_{2,5}$ are isomorphisms, because of Lemma 6.6. We consider the set

$$
\left\{(x, y): x \in C_{1}^{b(1)}, y \in C_{2}^{b(2)} \text { and } \pi_{2,5}(x)=\pi_{2,5}(y)\right\}
$$

which is nothing but the pullback of Diagram 7.1. One can check that this set equals $K_{b}$ since, as we observed before, the elements of $K$ are only subject to the equations coming from the cycles in $C_{1}$ and $C_{2}$. Since isomorphisms are stable 
under pullbacks, we obtain the following commutative diagram of isomorphisms:

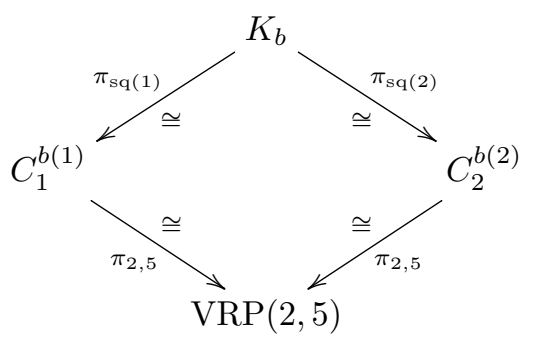

The composition $\pi_{2,5} \circ \pi_{\mathrm{sq}(1)}$ equals the projection $\pi_{2,5}: K_{b} \longrightarrow \operatorname{VRP}(2,5)$, and the same holds for every projection $\pi_{i, j}: K_{b} \longrightarrow \operatorname{VRP}(i, j)$, where $i$ and $j$ are neighboring links. So each of these maps is an isomorphism, proving our claim.

If now $G$ is constituted by more than two squares, then $K_{b}$ is obtained via an iteration of several pullbacks, and eventually we get a diagram of isomorphisms. Thus the claim holds also in this case. Since $K$ is covered by finitely many varieties of dimension one, it has dimension one. Moreover, each of the varieties $K_{b}$ is an irreducible component of $K$.

We are left to prove that $L$ realizes the motion $\phi$ induced by $P$. Similarly as we did in the proof of Proposition 7.1. one can show that image $(\phi) \subseteq \operatorname{RP}(1, n+1)$. Since $K$ is one-dimensional, also $\operatorname{RP}(1, n+1)$ is so; since image $(\phi)$ is one-dimensional, image $(\phi)$ is a component of $\operatorname{RP}(1, n+1)$. This concludes the proof.

Remark 7.7. As we mentioned in Section 1, the linkages constructed via our algorithms present the same issues as the ones produced by Kempe's procedure, namely the devices do not realize only the motion they are designed for, but also other ones. This is due to the fact that the elementary linkages produced by the flip procedure, commonly known as antiparallelograms, admit configuration spaces with more than one component (see Lemma 6.6). On the other hand, since antiparallelograms are among the linkages used also by Kempe's procedure, the techniques developed in [1] and [6, Section 3.2.2] can be applied also in our case to prevent this unwanted behavior.

Remark 7.8. Let $\phi$ be a rational motion given by $Z+\eta W \in \mathbb{K}[t]$. Our algorithm allows one to construct a linkage that generates $\phi$, using a motor that rotates at constant speed. For this purpose one needs two neighboring links whose relative position is a revolution with constant speed; such a motion is represented by a motion polynomial of the form $t \pm \imath+\eta w$. If $\imath$ or $-\imath$ is already a root of $Z$, then any linkage returned by ConstructStrongLinkage has the desired property. If not, then the linear polynomial $l$ in step 3 of this algorithm has to be chosen accordingly.

We now investigate how many links and joints the linkages created by our algorithm have. We consider the tasks of strongly realizing a given rational motion, and of drawing a given parametrized curve.

Proposition 7.9. Let $\phi$ be a rational motion given by a motion polynomial $P$ of degree $d$. Then there exists a linkage with at most $4 d+2$ links and $6 d+1$ strongly realizing $\phi$.

Proof. Apply ConstructStrongLinkage to $P$, and notice that the real polynomial $R$ produced by FactorMotionPolynomial has degree at most $d$. 
Proposition 7.10. Let $\varphi: \mathbb{R} \longrightarrow \mathbb{R}^{2}$ be the parametrization of a real bounded planar curve. Without loss of generality, suppose that

$$
\varphi(t)=\left(\frac{f(t)}{h(t)}, \frac{g(t)}{h(t)}\right)
$$

with $f, g, h \in \mathbb{R}[t]$, and $h$ monic, and $d:=\operatorname{deg} h>\max \{\operatorname{deg} f, \operatorname{deg} g\}$. There exists a linkage with at most $3 d+2$ links and $\frac{9}{2} d+1$ joints drawing the curve given by $\varphi$.

Proof. As discussed before Definition 5.13 the motion polynomial $P=h+\eta(f+\imath g)$ is bounded, and by Proposition 4.7 the orbit of the origin in $\mathbb{R}^{2}$ under the motion determined by $P$ is the image of $\varphi$. We could apply ConstructStrongLinkage to $P$, but we obtain a smaller linkage by applying this algorithm to $P^{\prime}=C P$, where $C \in \mathbb{C}[t]$ is any polynomial such that $C \bar{C}=h$ and $\operatorname{gcd}(C, \bar{C})=1$; such a $C$ exists because $h$ has no real roots. In fact, as discussed in Remark 4.9, the orbit of the origin under the motion given by $P^{\prime}$ is also the image of $\varphi$. Moreover, by Theorem 5.15, a motion polynomial $Z+\eta W$ is factorizable if the corresponding real polynomial $R$ equals 1 . This is the case if and only if $\operatorname{gcd}(Z, \bar{Z}) \mid W \bar{W}$; it is easy to check that this condition holds for $P^{\prime}$. Finally, the linkage obtained by applying ConstructStrongLinkage to $P^{\prime}$ is constituted by $3 d+2$ links and $\frac{9}{2} d+1$ joints, because the degree of $P^{\prime}$ is $\frac{3}{2} d$ - note that $d$ must be even.

Example 7.11. We illustrate the algorithm ConstructStrongLinkage with the example from Section 2, In contrast to Example 5.16, here we multiply the polynomial $P(t)=\left(t^{2}+1\right)+\eta(\imath t-2)$ by $C=t-\imath$, as prescribed by Proposition 7.10. Calling FactorMotionPolynomial with $C P$ as input, we get $R=1$ and obtain:

$$
C(t) \cdot P(t)=\left(t-\imath-\frac{1}{2} \eta \imath\right) \cdot\left(t-\imath+\frac{1}{2} \eta \imath\right) \cdot(t+\imath+\eta \imath) .
$$

Next we have to fix an $l \in \mathbb{K}$ such that the IFM condition holds; it turns out that $l=-\frac{9}{5} \imath-\frac{18}{35} \eta \imath=l_{1}$ is a good choice (which yields a linkage that is well suited for visualization, see Figure 22. Applying the flip procedure iteratively to this input data, one obtains the following:

$$
\begin{aligned}
& \widetilde{k}_{1}=\imath-\frac{13}{28} \eta \imath, \quad \widetilde{k}_{2}=\imath+\frac{5}{8} \eta \imath, \quad \widetilde{k}_{3}=-\imath-\frac{11}{56} \eta \imath, \\
& l_{2}=-\frac{9}{5} \imath+\frac{9}{20} \eta \imath, \quad l_{3}=-\frac{9}{5} \imath-\frac{27}{40} \eta \imath, \quad l_{4}=-\frac{9}{5} \imath-\frac{207}{140} \eta \imath .
\end{aligned}
$$

Now we can use these quantities to construct a linkage - whose graph is shown in Figure 8 - that draws an ellipse. Note that all linear polynomials appearing here are purely imaginary in their secondary parts. This implies that their fixed points are of the form $(x, 0)$, so that all joints are located on the horizontal axis when the linkage is in its initial position $(t=\infty)$; the same happens for $t=0$.

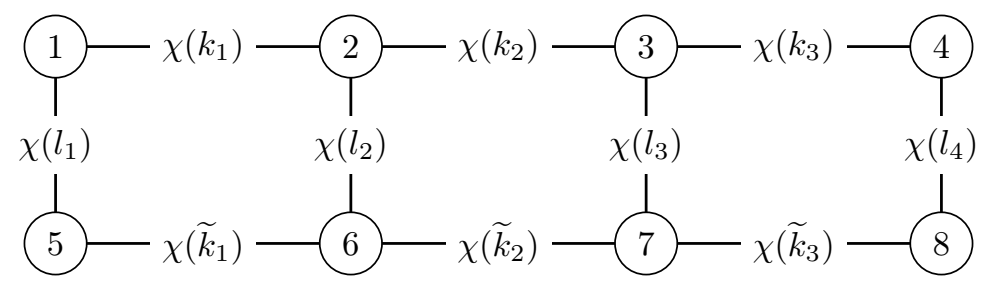

FIgURE 8. The link graph for the linkage drawing an ellipse. 


\section{Self-COlLisions}

A natural question that arises in the construction of a linkage is whether it can be physically realized such that no collisions between components of the linkage occur. More precisely, one asks whether there exists an assignment of $1,2, \ldots, n$ to the links such that for any $i<k<j$ for which $i$ and $j$ are neighboring links, the joint connecting $i$ and $j$ never overlaps with the link $k$. For general linkages it is a difficult problem to detect such collisions; see for example [5, Section 9.3 and Theorem 9.5.5]. However, it turns out that the same problem has a straightforward solution for linkages constructed by our algorithm, and the reason is that all joints in our case follow rational curves whose explicit parametrization is a direct byproduct of the algorithm ConstructStrongLinkage. Assuming that a link is realized as a collection of line segments connecting the (two or three) joints attached to it, a collision is described as follows: for some $i<k<j$ as above there exists a $t \in \mathbb{R} \cup\{\infty\}$ such that the position $\left(x_{1}(t), y_{1}(t)\right)$ of the joint $(i, j)$ lies on one of the line segments of link $k$; denote its endpoints by $\left(x_{2}(t), y_{2}(t)\right)$ and $\left(x_{3}(t), y_{3}(t)\right)$. Then a collision between joint $\{i, j\}$ and link $k$ happens if and only if the system

$$
\left\{\begin{array}{l}
x_{1}(t)=s \cdot x_{2}(t)+(1-s) \cdot x_{3}(t) \\
y_{1}(t)=s \cdot y_{2}(t)+(1-s) \cdot y_{3}(t)
\end{array}\right.
$$

has a solution for $0 \leq s \leq 1$ and $t \in \mathbb{R} \cup\{\infty\}$. In our construction, the coordinates $x_{i}, y_{i}$ are rational functions in $t$, so that the system 8.1 is equivalent to a bivariate polynomial system that can be easily solved. Note that we have to solve 8.1) for each admissible triple $i<k<j$. This way we can decide whether collisions occur or not, and if so, we get a precise description when and where they happen.

Example 8.1. Consider again the linkage constructed in Example 7.11, whose link graph is shown in Figure 8 . With the ordering $(5,1,6,2,7,8,4,3)$ of the links, we get two collisions, which both happen at $t=\infty$. Hence in principle we can trace, without disassembling the linkage, the full ellipse except a single point; see also the animations provided on our webpage [17].

At the same time, we want to mention a remarkable method for preventing collisions for planar linkages with a ladder-shaped link diagram as shown in Figure7. This method is based on designing the shape of some links and joints: we introduce three types of links (F-link, U-link, Z-link), and two types of joints (T-joint, Z-joint).

As before, all links are arranged in different layers, and w.l.o.g. we assume that these layers correspond to integer numbers. An F-link is located in a single layer, in other words, we associate one integer for each F-link. On the other hand, U-links and Z-links stretch across two, not necessarily neighboring, layers. Therefore, to such links we associate a pair of integers $(a, b)$ with $a<b$, which means that one part of the link is located in layer $a$ and the other part in layer $b$; the two parts are rigidly connected by a vertical rod. For two U-links with layers $\left(a_{1}, b_{1}\right) \neq\left(a_{2}, b_{2}\right)$, we prohibit the situations $a_{1}<a_{2}<b_{1}<b_{2}$ and $a_{2}<a_{1}<b_{2}<b_{1}$, which could yield a collision between these two U-links. For a Z-link on layers $(a, b)$ we impose the condition $b-a=2$, and the link located on layer $a+1$ (this will always be some part of a U-link) is connected with this Z-link by a revolute joint (which we call Z-joint) around its vertical rod. In contrast, a T-joint joins two links located on neighboring layers. These types of links and joints are illustrated in Figure 9 
Using the above design, one can check that collisions can only happen between links, not between links and joints. We argue now that even such collisions can be avoided. First, an F-link cannot collide with another F-link or a Z-link. Second, a Z-link cannot collide with another Z-link. Third, if we move the vertical rods of all U-links sufficiently far away, they do not collide with the F-links and Z-links. Fourth, the above conditions on the layers of U-links imply that two U-links can collide only if they occur in a nested way (e.g., $a_{1}<a_{2}<b_{2}<b_{1}$ ). Again, this can be avoided by moving the vertical rod of the outer U-link far enough away. Thus we can always make a collision-free design, by manipulating the shape of the U-links.

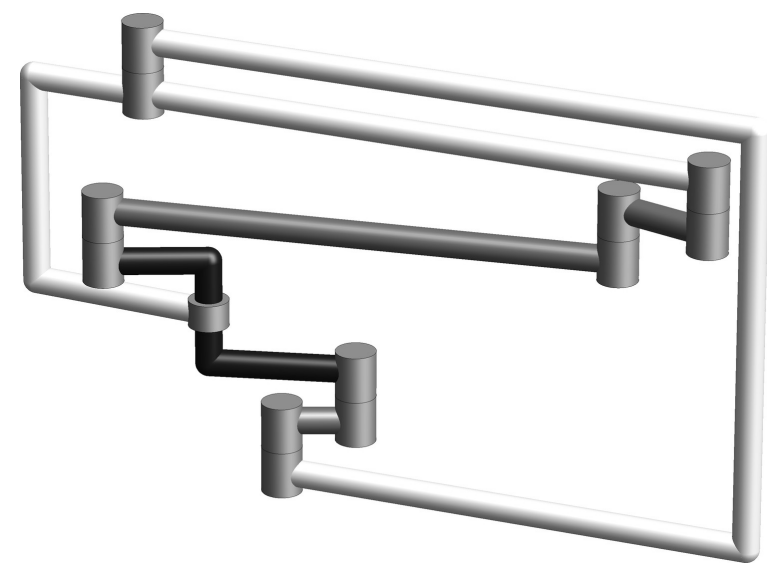

Figure 9. A linkage without self-collisions composed of three Flinks (gray), two U-links (white), and one Z-link (black)

It remains to argue that for a general ladder-shaped link diagram, as returned by our algorithm ConstructStrongLinkage and as displayed in Figure 7, we can find an assignment of link types and layers such that all the above conditions are fulfilled. One such assignment is depicted in Figure 10, it demonstrates that we can realize a motion - given by a factored motion polynomial of degree $n$ - by a collision-free linkage using $4 n+1$ layers.

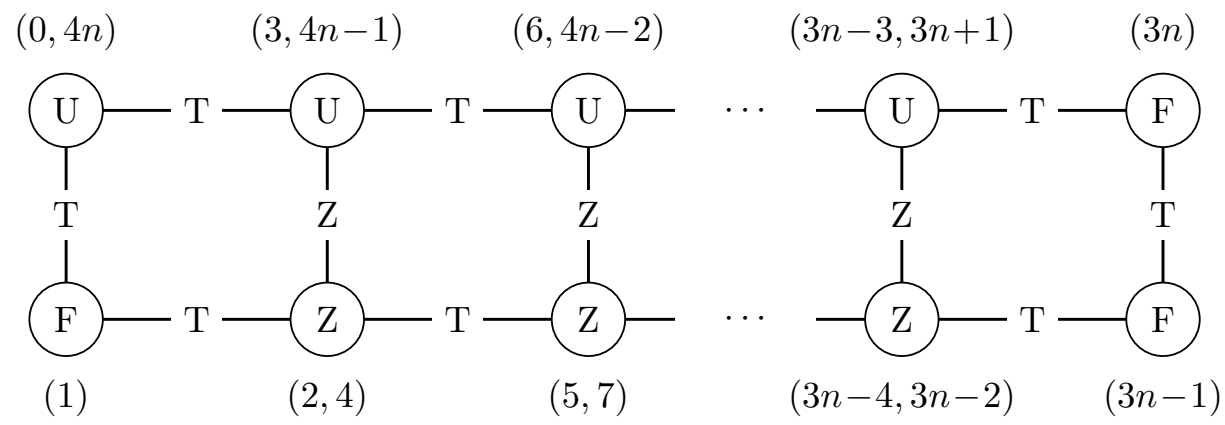

Figure 10. Assignment of joint types (T, Z), link types (F, U, Z) and layers (above and below the corresponding vertices) for realizing a linkage with ladder-shaped link graph without self-collisions. 


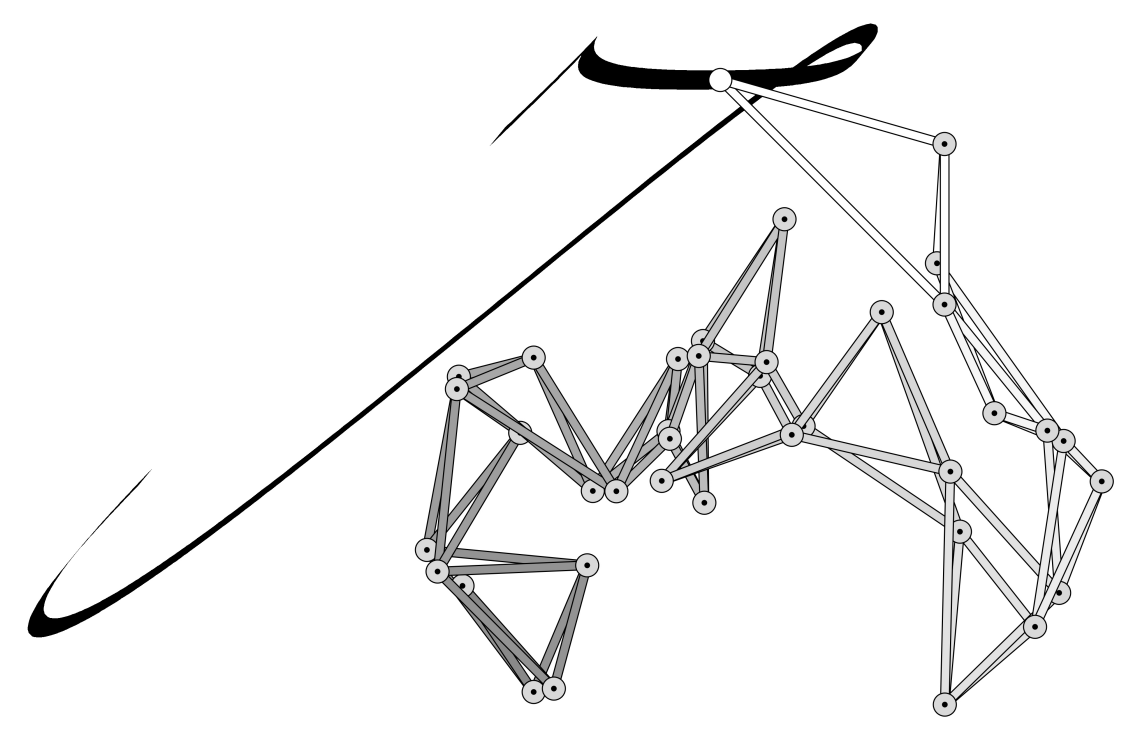

Figure 11. A rational curve approximating the "J" in John Hancock's signature and a linkage drawing it. The corresponding motion is a translation, which we visualize by using a quill pen whose shape is a line segment in direction $(5,6)$. The spatial arrangement of the links is indicated by hue: darker links lie below brighter ones.

\section{A SIGNING LINKAGE}

We conclude with an example in connection to a popular formulation of Kempe's Theorem, stating that "There is a linkage that signs your name". King [15, Corollary 1.3] attributes this formulation to William Thurston. However, as remarked by O'Rourke [23], it is very implausible that a concrete "signing linkage" has ever been constructed due to the complexity, in terms of links and joints, of the linkages produced following Kempe's procedure. As an example to support his claim, O'Rourke points out that already constructing a linkage drawing the "J" of John Hancock's famous signature (see Figure 12) would be very difficult.

We approximate the " $\mathrm{J}$ " by the rational curve given by the parametrization $(f / h, g / h)$, where

$$
\begin{aligned}
& f(t)=-321880 t^{5}-436132 t^{4}-237449 t^{3}-64488 t^{2}-8666 t-451, \\
& g(t)=-336018 t^{5}-472949 t^{4}-270569 t^{3}-78158 t^{2}-11325 t-651, \\
& h(t)=170\left(7225 t^{6}+13770 t^{5}+11187 t^{4}+4908 t^{3}+1219 t^{2}+162 t+9\right) .
\end{aligned}
$$

Our implementation [17 of the algorithm ConstructStrongLinkage produces a linkage with 26 links and 37 joints realizing a translational motion along this curve, see Figure 11, notice that these numbers are in accordance with Proposition 7.9. When this linkage draws the depicted "J", one encounters seven collisions.

\section{ACKNOWLEDGMENT}

We would like to thank the anonymous referee for helpful comments. 


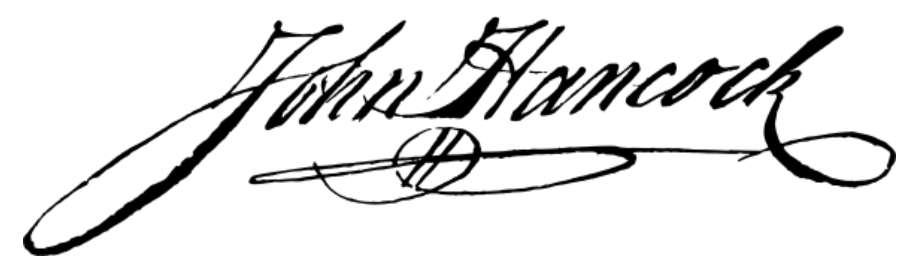

Figure 12. John Hancock's signature on the United States Declaration of Independence.

\section{REFERENCES}

[1] Timothy G. Abbott. Generalizations of Kempe's universality theorem. Master's thesis, Massachusetts Institute of Technology, 2008. Available at http://web.mit.edu/tabbott/www/ papers/mthesis.pdf

[2] Wilhelm Blaschke and Hans R. Müller. Ebene Kinematik. Verlag von R. Oldenbourg, München, 1956.

[3] Jacek Bochnak, Michel Coste, and Marie-Françoise Roy. Real algebraic geometry, volume 36 of Ergebnisse der Mathematik und ihrer Grenzgebiete (3). Springer-Verlag, Berlin, 1998.

[4] Oene Bottema and Bernard Roth. Theoretical Kinematics. North-Holland Publishing Company, 1979.

[5] Robert Connelly and Erik D. Demaine. Geometry and topology of polygonal linkages. In Jacob E. Goodman and Joseph O'Rourke, editors, Handbook of discrete and computational geometry, pages 197-218. Chapman \& Hall/CRC, Boca Raton, FL, second edition, 2004.

[6] Erik D. Demaine and Joseph O'Rourke. Geometric folding algorithms. Cambridge University Press, Cambridge, 2007.

[7] Xiao-Shan Gao and Chang-Cai Zhu. Automated generation of Kempe linkage and its complexity. Journal of Computer Science and Technology, 14(5):460-467, 1999.

[8] Xiao-Shan Gao, Chang-Cai Zhu, Shang-Ching Chou, and Jian-Xin Ge. Automated generation of Kempe linkages for algebraic curves and surfaces. Mechanism and Machine Theory, 36(9):1019-1033, 2001.

[9] Gábor Hegedüs, Josef Schicho, and Hans-Peter Schröcker. Construction of Overconstrained Linkages by Factorization of Rational Motions. In Jadran Lenarčič and Manfred Husty, editors, Latest Advances in Robot Kinematics, pages 213-220. Springer Netherlands, 2012.

[10] Gábor Hegedüs, Josef Schicho, and Hans-Peter Schröcker. Factorization of Rational Curves in the Study Quadric. Mechanism and Machine Theory, 69:142-152, 2013.

[11] Manfred L. Husty and Hans-Peter Schröcker. Kinematics and algebraic geometry. In J. Michael McCarthy, editor, 21st Century Kinematics, pages 85-123. Springer London, 2013.

[12] Denis Jordan and Marcel Steiner. Configuration spaces of mechanical linkages. Discrete 83 Computational Geometry, 22(2):297-315, 1999.

[13] Michael Kapovich and John J. Millson. Universality theorems for configuration spaces of planar linkages. Topology, 41(6):1051-1107, 2002.

[14] Alfred B. Kempe. On a General Method of describing Plane Curves of the nth degree by Linkwork. Proceedings of the London Mathematical Society, S1-7(1):213, 1876.

[15] Henry C. King. Planar linkages and algebraic sets. Turkish Journal of Mathematics, 23(1):33$56,1999$.

[16] Alexander Kobel. Automated generation of Kempe linkages for algebraic curves in a dynamic geometry system. Bachelor's thesis, University of Saarbrücken, 2008. Available at https: //people.mpi-inf.mpg.de/ akobel/publications/Kobel08-kempe-linkages.pdf.

[17] Christoph Koutschan. Mathematica package PlanarLinkages.m and electronic supplementary material for the paper "Planar linkages following a prescribed motion", 2015. Available at http://www.koutschan.de/data/link/

[18] Christian Krattenthaler. Advanced determinant calculus. Séminaire Lotharingien de Combinatoire, 42:1-67, 1999. Article B42q.

[19] Alain Lascoux and Piotr Pragacz. Jacobians of symmetric polynomials. Annals of Combinatorics, 6(2):169-172, 2002. 
[20] Henri Lebesgue. Leçons sur les Constructions Géométriques. Gauthier-Villars, Paris, 1950.

[21] Zijia Li, Josef Schicho, and Hans-Peter Schröcker. Factorization of motion polynomials. CoRR, abs/1502.07600, 2015. Available at http://arxiv.org/abs/1502.07600

[22] Joseph Malkevitch. Linkages: From fingers to robot arms. AMS Feature Column, September 2002. Available at http://www.ams .org/samplings/feature-column/fcarc-linkages1

[23] Joseph O'Rourke. How to fold it. Cambridge University Press, Cambridge, 2011.

[24] John Owen and Stephen Power. Continuous curves from infinite Kempe linkages. Bulletin of the London Mathematical Society, 41(6):1105-1111, 2009.

[25] Mark Plecnik, J. Michael McCarthy, and Charles W. Wampler. Kinematic Synthesis of a Watt I Six-Bar Linkage for Body Guidance. In Advances in Robot Kinematics, pages 317325. Springer International Publishing, 2014.

[26] Jon M. Selig. Geometric fundamentals of robotics. Monographs in Computer Science. Springer, New York, second edition, 2005.

(MG, CK, ZL, GR, NV) Johann Radon Institute for Computational and Applied Mathematics (RICAM), Austrian Academy of Sciences, Altenberger Strasse 69, 4040 Linz, Austria

(JS) Research Institute for Symbolic Computation (RISC), Johannes Kepler University, Altenberger Strasse 69, 4040 Linz, Austria

E-mail address: \{matteo.gallet, christoph.koutschan, zijia.li, georg.regensburger, josef.schicho, nelly.villamizar\}@ricam.oeaw.ac.at 